\title{
An elasto-plastic damage constitutive theory and its prediction of evolution of subsequent yield surfaces and elastic constants
}

\author{
Liu Fang*, Fu Qiang, Chen Cen, Liang Naigang \\ State Key Laboratory of Nonlinear Mechanics, Institute of Mechanics, Chinese Academy of Sciences, Beijing 100190, China
}

\section{A R T I C L E I N F O}

\section{Article history:}

Received 19 October 2010

Received in final revised form 28 December 2010

Available online 15 March 2011

\section{Keywords:}

Subsequent yield surface

Non-proportional loading

Finite deformation

Elasto-plastic damage constitutive relation

Component assembling model

\begin{abstract}
A B S T R A C T
Based on pair functional potentials, Cauchy-Born rule and slip mechanism, a material model assembling with spring-bundle components, a cubage component and slip components is established to describe the elasto-plastic damage constitutive relation under finite deformation. The expansion/shrink, translation and distortion of yield surfaces can be calculated based on the hardening rule and Bauschinger effect defined on the slip component level. Both kinematic and isotropic hardening are included. Numerical simulations and predictions under tension, torsion, and combined tension-torsion proportional/non-proportional loading are performed to obtain the evolution of subsequent yield surfaces and elastic constants and compare with two sets of experimental data in literature, one for a very low work hardening aluminum alloy $\mathrm{Al}$ 6061-T6511, and another for a very high work hardening aluminum alloy annealed $1100 \mathrm{Al}$. The feature of the yield surface in shape change, which presents a sharp front accompanied by a blunt rear under proportional loading, is described by the latent hardening and Bauschinger effect of slip components. Further, the evolution law of subsequent yield surfaces under different proportional loading paths is investigated in terms of their equivalence. The numerical simulations under non-proportional loading conditions for annealed $1100 \mathrm{Al}$ are performed, and the subsequent yield surfaces exhibit mixed cross effect because the kinematic hardening and isotropic hardening follow different evolution tendency when loading path changes. The results of non-proportional loading demonstrate that the present model has the ability to address the issue of complex loading due to the introduction of state variables on slip components. Moreover, as an elasto-plastic damage constitutive model, the present model can also reflect the variation of elastic constants through damage defined on the springbundle components.
\end{abstract}

(c) 2011 Elsevier Ltd. All rights reserved.

\section{Introduction}

Most sheet metal forming processes involve anisotropic behaviors with finite plastic deformation. Springback is a serious problem in sheet metal forming. It requires a comprehensive understanding of loading and unloading processes and determination of elastic constants to know the amount of springback for a given forming process. Therefore, it needs precise determination of subsequent yield surfaces. Researchers are focused on the evolution of yield surfaces either experimentally or analytically.

There are numerous investigations found in determination of yield surfaces for small (<5\%) prestrains including the ones by Naghdi et al. (1958), Ivey (1961), Michael and Findley (1976), Phillips and Ricciuti (1976), Moreton et al. (1981), Ascione

\footnotetext{
* Corresponding author. Tel.: +86 1082543926.

E-mail address: liufang@Inm.imech.ac.cn (F. Liu).
} 
et al. (1982), Phillips and Das (1985), Stout et al. (1985), Wu and Yeh (1991), Ishikawa (1997) and Gil et al. (1999). At the finite (>5\%) prestrains level, subsequent yield surfaces were determined by Mair and Pugh (1964), Williams and Svensson (1970, 1971), Shiratori et al. (1973), Tozawa (1978), Helling et al. (1986) and Khan and Wang (1988, 1993).

The expansion/shrink, translation, distortion, and rotation of the subsequent yield surfaces strongly depend on the materials, the loading paths, the probing paths, the magnitude of prestrains, as well as the definition of yielding. The definition of yielding is a key point for determining the subsequent yield surfaces. When the relatively large offset definition of yield is used, such as $0.2 \%$, the subsequent yield surfaces expand in size. When the relatively small offset definition of yield is used, such as 5-10 $\mu \varepsilon$, the subsequent yield surfaces will undergo significant kinematic hardening and distortion. In between these two extremes, investigators have used 10-1000 $\mu \varepsilon$ definition of yield where different mixes of kinematic and isotropic hardening are observed. Now with the development of experimental technique, a small offset definition of yield ( $<10 \mu \varepsilon)$ is often used by researchers.

Recently, Khan et al. (2009, 2010a,b) have done a series of experiments on the evolution of subsequent yield surfaces for two kinds of work hardening materials with finite strains. Besides determining the evolution of subsequent yield surfaces, Khan et al. (2009, 2010a,b) also studied the degradation of elastic constants with finite plastic deformation.

In order to study the evolution of subsequent yield surfaces, more advanced models should capture the distortion of the yield surface, as mechanical behavior and failure are very sensitive to small changes in the yield surface (Rousselier et al., 2009). Therefore, describing the evolution of subsequent yield surfaces accurately becomes a comprehensive examination of plasticity models on their predictability of mechanical behaviors in the plastic range. Numerous efforts have been made with the goal of more closely representing the behavior of subsequent yield surfaces, such as Rees (1984), Helling and Miller (1987, 1988), Gupta and Meyers (1992), Wegener and Schlegel (1996), Kowalsky et al. (1999), Kalidindi and Schoenfeld (2000), Francois (2001), Chiang et al. (2002), Mollica and Srinivasa (2002), Wu (2002, 2003b), Zattarin et al. (2004), Suprun (2006), Yeh and Lin (2006b) and Feigenbaum and Dafalias (2007, 2008). The analytical works can be categorized into two approaches (Wu et al., 1995), the algebraic approach and the geometric approach. In the algebraic approach, the yield function is generally defined in terms of an anisotropic material tensor, such as Ortiz and Popov (1983), Voyiadjis and Foroozesh (1990) and Feigenbaum and Dafalias $(2007,2008)$. In the geometric approach, the subsequent yield surface is described by a mapping technique that divides the yield surface into the forward and rear parts, such as Chiang et al. (2002), Yeh and Lin (2006a) and Fu et al. (2010).

On the other hand, the development of continuum damage mechanics expedites the elasto-plastic damage models. Most of the elasto-plastic damage constitutive models in the framework of continuum mechanics can be categorized into two approaches. One is based on the strain equivalence, stress equivalence or energy equivalence, such as Simo and Ju (1987), Lu and Chow (1990) and Souchet (2005), and another one is based on the fictitious undamaged configuration, such as Murakami (1988), Voyiadjis and Park (1999) and Brünig (2003). In the continuum model, the introduction of energy potential is essential. However, choosing appropriate energy potentials to account for the damage induced anisotropy and the issue of complex loading is a key problem for continuum models (Krajcinovic, 2000). Recently, an increasing number of researchers have concentrated efforts on developing continuum models incorporating information obtained from the microscopic level and therefore combining the advantages of continuum models and physical models.

Although some elasto-plastic damage models can describe the evolution of subsequent yield surfaces or elastic constants, the success and usefulness of these models are still limited due to the following reasons:

(1) The physical significance of model parameters. Some phenomenological models based on plastic potential which is expressed as a function of stress components and internal variables usually involve excessive model parameters which lack of physical significance.

(2) The distortion of subsequent yield surfaces. The common feature of the subsequent yield surfaces is a 'nose'. How to capture the distortion of subsequent yield surfaces is a key problem for most advanced models. It should be noticed that there are models with the capacity to describe the distortion of yield surfaces under proportional loading, such as Chiang et al. (2002), Yeh and Lin (2006a) and Feigenbaum and Dafalias (2007). However, the distortion of yield surface will complicate the evolution of yield surfaces, especially in the case of non-proportional loading and finite deformation.

(3) The evolution of subsequent yield surfaces under non-proportional loading. Due to lack of information about the plastic strain history, some yield surface models cannot be applied to non-proportional loading conditions, such as Voyiadjis and Foroozesh (1990), Chiang et al. (2002) and Yeh and Lin (2006a).

(4) The evolution of subsequent yield surfaces under finite deformation. While most experiments are conducted in the case of infinitesimal deformation, most of the plasticity models and investigations on evolution of yield surfaces are also limited to infinitesimal deformation. Wu (2003a) extended the classical flow theory of plasticity to the large strain range and used it to predict the initial and subsequent yield surfaces, in which mixed hardening was used and distortion of subsequent yield surfaces could not be considered.

(5) The degradation of elastic constants. While polycrystalline models usually focus on the plastic deformation induced anisotropy, most of them do not take the damage and damage induced anisotropy into account.

A physical mechanism based model called component assembling model has been formulated in small stain range by Deng et al. (2006, 2007) and Zhang et al. (2008). Liu et al. (2010) extends it into the condition of finite deformation. There 
are three kinds of components in the model, the spring-bundle, cubage and slip components. The spring-bundle and cubage components are abstracted from pair functional potentials, and the elasto-damage constitutive relation can be obtained by formulating the mechanical responses of these two kinds of components with damage reflected by the response function of spring-bundle components. Considering that slip is the main plastic deformation mechanism of polycrystalline metals, the slip component has been proposed by extending the slip system in single crystal to polycrystalline level. The plastic constitutive relation can be obtained by formulating the responses of slip components. Assembling these three kinds of components, based on the decomposition of total deformation into elastic deformation and plastic deformation, the elasto-plastic damage constitutive relation has been formed.

In this paper, the component assembling model established in the finite stain range is followed. The yield surface is determined by the positive and negative critical resolved shear stresses of all slip components which evolve under the hardening rule or Bauschinger effect. The self hardening, latent hardening and isotropic hardening are introduced in the hardening rule of the present model to reflect the different evolution of subsequent yield surfaces. Numerical simulations and predictions using the present model are performed to obtain the evolution of yield surfaces and elastic constants during tension, torsion, and combined tension-torsion proportional/non-proportional loading paths and compare with two sets of experimental data by Khan et al. (2009, 2010a), one for a very low work hardening aluminum alloy Al 6061-T6511, and another for a very high work hardening aluminum alloy annealed $1100 \mathrm{Al}$. The expansion/shrink, translation and distortion characterized by subsequent yield surfaces will be explained based on the hardening rule and Bauschinger effect defined on the slip component level. The variation of elastic constants will be determined based on the damage defined on the spring-bundle component level.

\section{Material model}

The component assembling model was proposed by Deng et al. (2006, 2007) in the infinitesimal deformation. Liu et al. (2010) extended it into the condition of finite deformation. In this investigation, the derivation process of the material model under finite deformation is followed.

\subsection{Elasto-damage constitutive relation}

Pair functional potentials describe the energy of materials in terms of atomic bonds and atom embedding interactions. According to the theory of pair functional potentials, the total energy of materials is divided into two parts. One part is pair potential $\varphi\left(r^{(\alpha, \beta)}\right)$ representing the Coulombic repulsive interactions among atomic nucleus and depending only on the atomic spacing $r^{(\alpha, \beta)}$. The other is embedding potential $F\left(\rho^{(\alpha)}\right)$ modeling the interaction of that atoms embedded into the "electron sea”, which is a function of the local electron density $\rho^{(\alpha)}$. The total energy is expressed as

$$
E_{\text {tot }}=E_{C}+E_{V}=\frac{1}{2} \sum_{(\alpha, \beta)} \phi\left(r^{(\alpha, \beta)}\right)+\sum_{\alpha} F\left(\rho^{(\alpha)}\right)
$$

The deformation of the atomic bond can be defined as

$$
\lambda^{(\alpha, \beta)}=\ln \frac{r^{(\alpha, \beta)}}{r_{0}^{(\alpha, \beta)}}
$$

where $r_{0}^{(\alpha, \beta)}$ is the initial distance between atoms $\alpha$ and $\beta$. Using the deformation rate of atomic bond, the time rate of pair potential can be expressed as

$$
\dot{E}_{C}=\frac{1}{2} \sum_{(\alpha, \beta)} \tilde{f}\left(\lambda^{(\alpha, \beta)}\right) \dot{\lambda}^{(\alpha, \beta)}
$$

where $\tilde{f}\left(\lambda^{(\alpha, \beta)}\right)$ is regarded as the atomic bonding force response function,

$$
\tilde{f}\left(\lambda^{(\alpha, \beta)}\right)=\frac{\partial \phi}{\partial \lambda^{(\alpha, \beta)}}=\frac{\partial \phi}{\partial r^{(\alpha, \beta)}} r_{0}^{(\alpha, \beta)} e^{\lambda^{(\alpha, \beta)}}
$$

Analogously, the volumetric deformation of electron gas can be defined as

$$
\theta^{(\alpha)}=\ln \frac{\rho_{0}^{(\alpha)}}{\rho^{(\alpha)}}
$$

where $\rho_{0}^{(\alpha)}$ is the initial electron density at atom $\alpha$. Using the volumetric deformation of electron gas, the time rate of embedding potential is given by

$$
\dot{E}_{V}=\sum_{\alpha} \tilde{p}\left(\theta^{(\alpha)}\right) \dot{\theta}^{(\alpha)}
$$

where $\tilde{p}\left(\theta^{(\alpha)}\right)$ is regarded as the volumetric force response function,

$$
\tilde{p}\left(\theta^{(\alpha)}\right)=\frac{\partial F}{\partial \theta^{(\alpha)}}=-\frac{\partial F}{\partial \rho^{(\alpha)}} \rho_{0}^{(\alpha)} e^{-\theta^{(\alpha)}}
$$


By combining Eqs. (3) and (6), the time rate of total energy is

$$
\dot{E}_{\text {tot }}=\frac{1}{2} \sum_{(\alpha, \beta)} \tilde{f}\left(\lambda^{(\alpha, \beta)}\right) \dot{\lambda}^{(\alpha, \beta)}+\sum_{\alpha} \tilde{p}\left(\theta^{(\alpha)}\right) \dot{\theta}^{(\alpha)}
$$

We define the initial undeformed state as reference configuration and the deformed state as current configuration. Considering a material element with unit volume in reference configuration, the deformation work is expressed as

$$
W^{e}=\left.E_{\text {tot }}\right|_{\text {def }}-\left.E_{\text {tot }}\right|_{\text {undef }}
$$

where $\left.E_{\text {tot }}\right|_{\text {def }}$ and $\left.E_{\text {tot }}\right|_{\text {undef }}$ denote the total energy of current and reference configurations respectively. Herein, $W^{e}$ refers to the elastic part, and the decomposition of the total deformation will be presented in Section 2.2. Using Eq. (8), the deformation work rate is given by

$$
\dot{W}^{e}=\left.\dot{E}_{t o t}\right|_{d e f}=\dot{W}_{C}^{e}+\dot{W}_{V}^{e}=\frac{1}{2} \sum_{(\alpha, \beta)} \tilde{f}\left(\lambda^{(\alpha, \beta)}\right) \dot{\lambda}^{(\alpha, \beta)}+\sum_{\alpha} \tilde{p}\left(\theta^{(\alpha)}\right) \dot{\theta}^{(\alpha)}
$$

It can be seen from Eq. (10) that the calculation of rate of pair potential $\dot{W}_{C}^{e}$ needs to run over all atomic bonds and that of rate of embedding potential $\dot{W}_{V}^{e}$ needs to run over all atoms. Since the material element consists of a huge number of atoms, the number of kinematic degree of freedom is too large.

For solid materials, Cauchy-Born rule (Ericksen, 1984) builds a bridge between microscopic movements and macroscopic deformations. It asserts that atoms in a material subject to a homogeneous deformation move according to a single mapping from the undeformed to the deformed configuration.

As to the atomic bond between atoms $\alpha$ and $\beta$, the deformation of this atomic bond is $\lambda^{(\alpha, \beta)}$. Let $n$ be the direction of atomic bond and $\lambda^{(\mathbf{n})}$ be the macroscopic deformation in this direction. Then, according to Cauchy-Born rule, it holds that

$$
\lambda^{(\alpha, \beta)}=\lambda^{(\mathbf{n})}
$$

Above equation indicates that the atomic bonds in the same direction follow the same deformation history which is identical to the macroscopic deformation in this direction. Therefore, it is feasible to group atomic bonds according to their directions.

Considering a unit volume element in the reference configuration, the deformations of atomic bonds in the neighborhood of a little solid angle $(\Delta \Omega)_{0}^{(s)}$ around $\mathbf{N}^{(s)}$ direction are assumed to be the same as the deformation in the $\mathbf{N}^{(s)}$ direction. The contributions of atomic bonds in this neighborhood to the deformation work rate is

$$
\dot{W}_{C}^{e(s)}=\frac{1}{2} \sum_{\mathbf{N}^{(\alpha, \beta)} / / \mathbf{N}^{(s)}} \tilde{f}\left(\lambda^{(\alpha, \beta)}\right) \dot{\lambda}^{(\alpha, \beta)}=f\left(\lambda^{(s)}\right) \dot{\lambda}^{(s)}
$$

where the sum symbol denotes summing over atomic bonds in $(\Delta \Omega)_{0}^{(s)}$ neighborhood of $\mathbf{N}^{(s)}$ direction, and $f\left(\lambda^{(s)}\right)$ denotes the summation of atomic bonding forces in this neighborhood,

$$
f\left(\lambda^{(s)}\right)=\frac{1}{2} \sum_{\mathbf{N}^{(\alpha, \beta)} / / \mathbf{N}^{(s)}} \tilde{f}\left(\lambda^{(\alpha, \beta)}\right)=\frac{1}{2} \sum_{\mathbf{N}^{(\alpha, \beta)} / / \mathbf{N}^{(s)}} \frac{\partial \phi}{\partial r^{(\alpha, \beta)}} r_{0}^{(\alpha, \beta)} e^{\lambda^{(\alpha, \beta)}}
$$

Therefore, the atomic bonds in the $(\Delta \Omega)_{0}^{(s)}$ neighborhood of $\mathbf{N}^{(s)}$ direction follow the same deformation history and can be grouped together. All the atomic bonds in the $(\Delta \Omega)_{0}^{(s)}$ neighborhood of $\mathbf{N}^{(s)}$ direction can be represented by a spring-bundle component. The summation of atomic bonding forces in this neighborhood corresponds to the force of this spring-bundle component, and the deformation of atomic bonds corresponds to the deformation of this spring-bundle component. Since the pair potential changes with atomic distances only, spring-bundle component only bears tension or compression being decomposed into the direction of this component. Its force response function $f\left(\lambda^{(s)}\right)$ is expressed by Eq. (13).

All directions in space can be divided into neighborhoods of $n$ discrete representative directions, and each direction corresponds to a spring-bundle component. Consequently, the part of the deformation work rate $\dot{W}_{C}^{e}$ originating from pair potential can be expressed as the summation of contributions of all spring-bundle components,

$$
\dot{W}_{C}^{e}=\sum_{s=1}^{n} \dot{W}_{C}^{e(s)}=\sum_{s=1}^{n} f\left(\lambda^{(s)}\right) \dot{\lambda}^{(s)}
$$

The dimension of material element is much larger than that of atom with statistically uniform property. It is assumed that the electron density is homogeneous within the material element. Accordingly, the volumetric deformation of electron gas and volumetric force response function are also the same for every atom. In continuum deformation field, the volumetric deformation is taken as $\theta$. According to Cauchy-Born rule, there is

$$
\theta^{(\alpha)}=\theta
$$

Therefore, the other part of the deformation work rate $\dot{W}_{V}^{e}$ originating from embedding potential can be simplified as

$$
\dot{W}_{V}^{e}=\sum_{\alpha} \tilde{p}\left(\theta^{(\alpha)}\right) \dot{\theta}^{(\alpha)}=p(\theta) \dot{\theta}
$$


where $p(\theta)$ denotes the summation of volumetric forces in unit reference volume,

$$
p(\theta)=\sum_{\alpha} \tilde{p}\left(\theta^{(\alpha)}\right)=-\sum_{\alpha} \frac{\partial F}{\partial \rho^{(\alpha)}} \rho_{0}^{(\alpha)} e^{-\theta^{(\alpha)}}
$$

Consequently, $\dot{W}_{V}^{e}$ has only one generalized degree of freedom $\theta$, and the atom embedding interaction can be represented by a cubage component. The deformation of the cubage component is volumetric deformation $\theta$, and its force $p(\theta)$ is expressed by Eq. (17). Since the embedding potential only responds to the volumetric deformation, the cubage component only bears hydrostatic stress.

By combining Eqs. (14) and (16), the total deformation work rate can be described as

$$
\dot{W}^{e}=\sum_{s=1}^{n} f\left(\lambda^{(s)}\right) \dot{\lambda}^{(s)}+p(\theta) \dot{\theta}
$$

Thereby, a material model composed of spring-bundle components and a cubage component is established.

Once the deformation work rate is obtained, the derivation of constitutive equations will follow the similar derivation process of traditional continuum models. In Eq. (18), the deformation work rate is described by the deformation rates of spring-bundle and cubage components. The relations between the deformation rates of two kinds of components and the macroscopic deformation rate will be set up below, so that the deformation work rate can be expressed as a function of the macroscopic deformation rate.

Similar to the deformation of atomic bond, we define the deformation of material line element in the direction of $\mathbf{N}$ as

$$
\lambda=\ln l=\frac{1}{2} \ln (\mathbf{n} \cdot \mathbf{n})=\frac{1}{2} \ln \left((\mathbf{N} \otimes \mathbf{N}):\left(\left(\mathbf{F}^{e}\right)^{T} \cdot \mathbf{F}^{e}\right)\right)
$$

where $l$ denotes the length of material line element after deformation, $\mathbf{n}$ represents the unit vector $\mathbf{N}$ after deformation, and $\mathbf{F}^{e}$ refers to the elastic deformation gradient. The time rate of $\lambda$ is

$$
\dot{\lambda}=\frac{(\mathbf{n} \cdot \mathbf{n})^{\cdot}}{2 \mathbf{n} \cdot \mathbf{n}}=e^{-2 \lambda}(\mathbf{n} \otimes \mathbf{n}): \mathbf{L}^{e}=e^{-2 \lambda}(\mathbf{n} \otimes \mathbf{n}): \mathbf{D}^{e}
$$

where $\mathbf{L}^{e}$ is the velocity gradient, and $\mathbf{D}^{e}$ is the symmetric rate of stretching tensor.

Similar to the volumetric deformation of electron gas, we define the volumetric deformation of material volume element as

$$
\theta=\ln J=\ln \left(\operatorname{det} \mathbf{F}^{e}\right)
$$

where $J$ is the ratio of volume in the current configuration to that in the reference configuration. Then, the time rate of $\theta$ is

$$
\dot{\theta}=\frac{1}{J} \dot{J}=\frac{1}{J}\left(J \mathbf{I}: \mathbf{D}^{e}\right)=\mathbf{I}: \mathbf{D}^{e}
$$

where I denotes the second-order identity tensor.

The deformation rates of spring-bundle and cubage components can be expressed by Eqs. (20) and (21), respectively. Then, the deformation work rate can be expressed as

$$
\dot{W}^{e}=\sum_{s=1}^{n} f(\lambda) e^{-2 \lambda}(\mathbf{n} \otimes \mathbf{n}): \mathbf{D}^{e}+p(\theta) \mathbf{I}: \mathbf{D}^{e}
$$

For the sake of clarity, the superscript(s) of spring-bundle components is omitted.

Let $\boldsymbol{\sigma}$ denote the Cauchy stress, the work rate per unit reference volume can be expressed as

$$
\dot{W}^{e}=J \boldsymbol{\sigma}: \mathbf{D}^{e}
$$

According to Eqs. (23) and (24), the Cauchy stress should be expressed as

$$
\boldsymbol{\sigma}=\frac{1}{J}\left(\sum_{s=1}^{n} f(\lambda) e^{-2 \lambda}(\mathbf{n} \otimes \mathbf{n})+p(\theta) \mathbf{I}\right)
$$

The above expression sets up the relations between the forces of spring-bundle and cubage components with the macroscopic stress field. On the other hand, the deformations of these two kinds of components are related to the macroscopic deformation field by Eqs. (19) and (21). Therefore, it is feasible to transform the strain-stress relations in 6-dimensional space to the force response functions of two kinds of components with only one degree of freedom. That is to say, the complex macroscopic response of materials can be obtained through assembling the simple responses of two kinds of components.

Based on the relation between Kirchhoff stress $\tau$ and Cauchy stress $\boldsymbol{\sigma}$, the Kirchhoff stress is given by

$$
\boldsymbol{\tau}=J \boldsymbol{\sigma}=\sum_{s=1}^{n} f(\lambda) e^{-2 \lambda}(\mathbf{n} \otimes \mathbf{n})+p(\theta) \mathbf{I}
$$


Correspondingly, the Jaumann rate of Kirchhoff stress is expressed as

$$
\begin{aligned}
\stackrel{\nabla}{\tau}\left(\mathbf{W}^{e}\right)= & \dot{\boldsymbol{\tau}}+\boldsymbol{\tau} \cdot \mathbf{W}^{e}-\mathbf{W}^{e} \cdot \boldsymbol{\tau}=\sum_{s=1}^{n}\left(f^{\prime}(\lambda)-2 f(\lambda)\right) e^{-4 \lambda}\left((\mathbf{n} \otimes \mathbf{n}): \mathbf{D}^{e}\right)(\mathbf{n} \otimes \mathbf{n}) \\
& +\sum_{s=1}^{n} f(\lambda) e^{-2 \lambda}\left(\mathbf{D}^{e} \cdot(\mathbf{n} \otimes \mathbf{n})+(\mathbf{n} \otimes \mathbf{n}) \cdot \mathbf{D}^{e}\right)+p^{\prime}(\theta)\left(\mathbf{I}: \mathbf{D}^{e}\right) \mathbf{I}
\end{aligned}
$$

The Jaumann rate of Kirchhoff stress is objective, so that the constitutive equation can be written as

$$
{\stackrel{\nabla}{\boldsymbol{\tau}_{\left(\mathbf{W}^{e}\right)}}}=\mathbf{C}^{e}: \mathbf{D}^{e}
$$

where $\mathbf{C}^{e}$ is the tangent stiffness tensor. Its components are expressed as

$$
C_{i j k l}^{e}=\sum_{s=1}^{n}\left(f^{\prime}(\lambda)-2 f(\lambda)\right) e^{-4 \lambda} n_{i} n_{j} n_{k} n_{l}+\frac{1}{2} \sum_{s=1}^{n} f(\lambda) e^{-2 \lambda}\left(n_{j} n_{l} \delta_{i k}+n_{i} n_{k} \delta_{j l}+n_{j} n_{k} \delta_{i l}+n_{i} n_{l} \delta_{j k}\right)+p^{\prime}(\theta) \delta_{i j} \delta_{k l}
$$

In microscopic level, damage and fracture are related to the breaking of atomic bonds which are represented by the spring-bundle component. When atomic debonding emerges, the corresponding spring-bundle component will change its mechanical properties. Therefore, the Eqs. (28) and (29) describe the elasto-damage constitutive relation of materials.

\subsection{Elasto-plastic damage constitutive relation}

The above elasto-damage constitutive relation is based on pair functional potentials. However, due to not changing the length of atomic bonds, atomic movement perpendicular to atomic bond, such as dislocation introduced by Taylor (1934), changes the lattice structure and therefore the energy of materials hardly. Therefore, the pair functional potentials cannot reflect the energy change in the process of dislocation slip.

As the main plastic deformation mechanism of most metals, dislocation slip is investigated both experimentally and theoretically. The well-known experimental evidence is that the earliest-appearing slip lines in polycrystalline metals are always parallel to the plane with the largest shear stress exerted. Therefore, in polycrystalline aggregate, a close approximation was obtained by Martin (1975) to assume that the slip can occur along all planes in a homogeneously loaded element. In the previous works of Deng et al. (2007), crystal plasticity is extended to polycrystalline, and the slip component is proposed to describe the macro characteristics of slip systems in different crystals which have the same orientation tensor. Analogous simplification can be traced back to the 1940s when Batdorf and Budiansky (1949) proposed a simple slip theory, in which each grain has only one slip system. Martin (1975) assumed that slip can occur along all planes in materials. Wang (1991) proposed a macro slip theory, in which four independent slip systems are used for polycrystalline solids. Liang et al. (1998) proposed a meso elastoplastic constitutive model based on equivalent slip systems, in which the tiny slip systems distributing randomly between crystal slices in micro-grains or on grain boundaries are replaced by macro equivalent slip systems. Khan and Cheng (1996) proposed an anisotropic elastic-plastic constitutive model in which slip systems are distributed randomly. In the previous works of Deng et al. (2007), the slip component has been proposed to describe plastic deformation in the case of infinitesimal deformation. Liu et al. (2010) describes the response of slip component under finite deformation similar to crystal plasticity (Asaro, 1983). In this section, the response of slip component is also described within the framework of finite deformation, and then the corresponding plastic constitutive relation in the case of finite deformation is obtained.

In a crystalline solid, an increment of deformation is imagined to occur in two steps. The first, starting from the reference state, occurs by a process of simple shears on slip systems. The deformation gradient in this step is called plastic deformation gradient and denoted by $\mathbf{F}^{p}$. Plastic deformation via dislocation slip deforms and rotates the material, but does not affect the lattice structure. Following this is a process of lattice deformation, the deformation gradient in this step is called elastic deformation gradient and denoted by $\mathbf{F}^{e}$. The material and lattice deform and rotate together under $\mathbf{F}^{e}$. The basic kinematic scheme is shown in Fig. 1. Thus, the deformation gradient is decomposed as

$$
\mathbf{F}=\mathbf{F}^{e} \cdot \mathbf{F}^{p}
$$

More sophisticated kinematic approaches are investigated by Voyiadjis and Park (1999), Brünig (2003). It should be noticed that though the above simple decomposition is used in the present paper, it can be extended to a more sophisticated approach if the elastic deformation is further decomposed into an elastic part and a damage part. The further decomposition will not be discussed here in detail.

The response of plastic deformation based on slip components is similar to that of crystal plasticity theory. Let $\mathbf{S}^{(\alpha)}$ and $\mathbf{N}^{(\alpha)}$ be the unit vectors in the slip direction and the normal of slip plane on slip component $\alpha$ in the reference configuration. Since the plastic deformation does not change the structure of lattice, the slip direction and normal of slip plane are not changed after plastic deformation, as shown in Fig. 1. After elastic deformation, the slip direction and the corresponding normal on slip component $\alpha$ become to $\mathbf{s}^{(\alpha)}$ and $\mathbf{n}^{(\alpha)}$ which satisfy

$$
\mathbf{S}^{(\alpha)}=\mathbf{F}^{e} \cdot \mathbf{S}^{(\alpha)}
$$




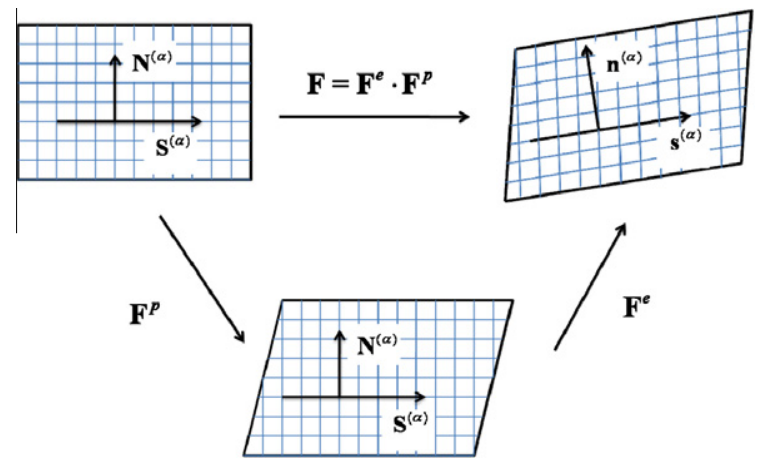

Fig. 1. Kinematic model of elasto-plastic deformation in crystalline media.

and

$$
\mathbf{n}^{(\alpha)}=\mathbf{N}^{(\alpha)} \cdot\left(\mathbf{F}^{e}\right)^{-1}
$$

respectively.Accordingly, we introduce second-order symmetric tensor $\mathbf{P}^{(\alpha)}$ and antisymmetric tensor $\mathbf{Q}^{(\alpha)}$ as

$$
\mathbf{P}^{(\alpha)}=\frac{1}{2}\left(\mathbf{s}^{(\alpha)} \otimes \mathbf{n}^{(\alpha)}+\mathbf{n}^{(\alpha)} \otimes \mathbf{s}^{(\alpha)}\right)
$$

and

$$
\mathbf{Q}^{(\alpha)}=\frac{1}{2}\left(\mathbf{s}^{(\alpha)} \otimes \mathbf{n}^{(\alpha)}-\mathbf{n}^{(\alpha)} \otimes \mathbf{S}^{(\alpha)}\right)
$$

respectively.

The kinematic and kinetic variables of the slip component $\alpha$ are shearing deformation $\gamma^{(\alpha)}$ and resolved shear stress $\tau^{(\alpha)}$ respectively. The resolved shear stress is computed using Schmid law,

$$
\tau^{(\alpha)}=J \boldsymbol{\sigma}: \mathbf{P}^{(\alpha)}=\tau: \mathbf{P}^{(\alpha)}
$$

where $\boldsymbol{\sigma}$ is Cauchy stress and $\tau$ is Kirchhoff stress. The time rate of $\tau^{(\alpha)}$ is

$$
\dot{\tau}^{(\alpha)}=\dot{\tau}: \mathbf{P}^{(\alpha)}+\left(\boldsymbol{\tau} \cdot \mathbf{W}^{e}-\mathbf{W}^{e} \cdot \boldsymbol{\tau}\right): \mathbf{P}^{(\alpha)}+\left(\mathbf{Q}^{(\alpha)} \cdot \tau-\tau \cdot \mathbf{Q}^{(\alpha)}\right): \mathbf{D}^{e}
$$

In the theory of crystal plasticity, the symmetric and antisymmetric parts of plastic velocity gradient have following relationship with second-order tensors $\mathbf{P}^{(\alpha)}$ and $\mathbf{Q}^{(\alpha)}$,

$$
\begin{aligned}
& \mathbf{D}^{p}=\sum_{\alpha=1}^{m} \dot{\gamma}^{(\alpha)} \mathbf{P}^{(\alpha)} \\
& \mathbf{W}^{p}=\sum_{\alpha=1}^{m} \dot{\gamma}^{(\alpha)} \mathbf{Q}^{(\alpha)}
\end{aligned}
$$

where $\dot{\gamma}^{(\alpha)}$ is the slip shearing deformation rate on slip component $\alpha$. Substituting Eq. (37) into Eq. (36), according to the elasto-damage constitutive equation (28), Eq. (36) can be expressed as

$$
\dot{\tau}^{(\alpha)}=\left(\mathbf{P}^{(\alpha)}: \mathbf{C}^{e}+\mathbf{Q}^{(\alpha)} \cdot \tau-\tau \cdot \mathbf{Q}^{(\alpha)}\right): \mathbf{D}^{e}=\lambda^{(\alpha)}:\left(\mathbf{D}-\sum_{\alpha=1}^{m} \dot{\gamma}^{(\alpha)} \mathbf{P}^{(\alpha)}\right)
$$

where

$$
\lambda^{(\alpha)}=\mathbf{P}^{(\alpha)}: \mathbf{C}^{e}+\mathbf{Q}^{(\alpha)} \cdot \tau-\tau \cdot \mathbf{Q}^{(\alpha)}
$$

According to the generalized Schmid's law, the resolved shear stress $\tau^{(\alpha)}$ of the $\alpha$ slip component must reach its critical value $\tau_{ \pm c r}^{(\alpha)}$, the slip component is said to be the potentially active or critical component. Otherwise, it is said to be the noncritical component. For the slip component $\alpha$ remaining active, $\tau^{(\alpha)}$ must increase to and remain at the critical value $\tau_{ \pm c r}^{(\alpha)}$,

$$
\begin{cases}\dot{\tau}_{+c r}^{(\alpha)}=\dot{\tau}^{(\alpha)}, \dot{\gamma}^{(\alpha)}>0 & \text { if } \dot{\tau}^{(\alpha)}>0 \\ \dot{\tau}_{-c r}^{(\alpha)}=\dot{\tau}^{(\alpha)}, \dot{\gamma}^{(\alpha)}<0 & \text { if } \dot{\tau}^{(\alpha)}<0\end{cases}
$$

For a noncritical or inactive slip component $\alpha$, there is

$$
\tau_{-c r}^{(\alpha)}<\tau^{(\alpha)}<\tau_{+c r}^{(\alpha)}
$$


and

$$
\dot{\gamma}^{(\alpha)}=0
$$

During the slipping process, considering latent hardening, it is assumed that the rate of critical resolved shear stress is linearly related to the shearing deformation rates on slip components. If only kinematic hardening is considered, the rate of critical resolved shear stress on the slip component $\alpha$ is given by

$$
\begin{cases}\dot{\tau}_{+c r}^{(\alpha)}=\sum_{\beta=1}^{m} h_{\alpha \beta} \dot{\gamma}^{(\beta)}, \dot{\tau}_{-c r}^{(\alpha)}=b \dot{\tau}_{+c r}^{(\alpha)} & \sum_{\beta=1}^{m} h_{\alpha \beta} \dot{\gamma}^{(\beta)}>0 \\ \dot{\tau}_{-c r}^{(\alpha)}=\sum_{\beta=1}^{m} h_{\alpha \beta} \dot{\gamma}^{(\beta)}, \dot{\tau}_{+c r}^{(\alpha)}=b \dot{\tau}_{-c r}^{(\alpha)} & \sum_{\beta=1}^{m} h_{\alpha \beta} \dot{\gamma}^{(\beta)}<0\end{cases}
$$

Herein $h_{\alpha \beta}$ is the hardening modulus matrix, $\alpha=\beta$ denotes the self-hardening moduli and $\alpha \neq \beta$ denotes the latent hardening moduli. $b$ is the Bauschinger effect parameter. When $\sum_{\beta=1}^{m} h_{\alpha \beta} \dot{\gamma}^{(\beta)}>0$, the positive critical resolved shear stress changes due to hardening, and the negative one changes through Bauschinger effect, i.e. $\dot{\tau}_{-c r}^{(\alpha)}=b \dot{\tau}_{+c r}^{(\alpha)}$, and vice versa.

For the active slip component, Eq. (41) holds. Substituting Eqs. (39) and (44) into Eq. (41), we obtain

$$
\boldsymbol{\lambda}^{(\alpha)}:\left(\mathbf{D}-\sum_{\alpha=1}^{m} \dot{\gamma}^{(\alpha)} \mathbf{P}^{(\alpha)}\right)=\sum_{\beta=1}^{m} h_{\alpha \beta} \dot{\gamma}^{(\beta)}
$$

Then,

$$
\lambda^{(\alpha)}: \mathbf{D}=\sum_{\beta=1}^{m}\left(h_{\alpha \beta}+\lambda^{(\alpha)}: \mathbf{P}^{(\beta)}\right) \dot{\gamma}^{(\beta)}=\sum_{\beta=1}^{m} g_{\alpha \beta} \dot{\gamma}^{(\beta)}
$$

where

$$
g_{\alpha \beta}=h_{\alpha \beta}+\lambda^{(\alpha)}: \mathbf{P}^{(\beta)}
$$

Consequently, for an active slip component $\alpha$, the shearing deformation rate can be expressed as

$$
\dot{\gamma}^{(\alpha)}=\sum_{\beta=1}^{m}\left(g_{\alpha \beta}\right)^{-1} \lambda^{(\beta)}: \mathbf{D}
$$

Using Eqs. (28) and (48), the Jaumann rate of Kirchhoff stress based on the continuum spin $\mathbf{W}$ is

$$
\begin{aligned}
& \stackrel{\nabla}{\tau}=\dot{\boldsymbol{\tau}}+\boldsymbol{\tau} \cdot \mathbf{W}-\mathbf{W} \cdot \boldsymbol{\tau}=\dot{\boldsymbol{\tau}}+\boldsymbol{\tau} \cdot \mathbf{W}^{e}-\mathbf{W}^{e} \cdot \boldsymbol{\tau}+\boldsymbol{\tau} \cdot \mathbf{W}^{p}-\mathbf{W}^{p} \cdot \boldsymbol{\tau}=\mathbf{C}^{e}:\left(\mathbf{D}-\mathbf{D}^{p}\right)-\sum_{\alpha=1}^{m} \dot{\gamma}^{(\alpha)}\left(\mathbf{Q}^{(\alpha)} \cdot \boldsymbol{\tau}-\boldsymbol{\tau} \cdot \mathbf{Q}^{(\alpha)}\right)=\mathbf{C}^{e} \\
& \quad: \mathbf{D}-\sum_{\alpha=1}^{m} \dot{\gamma}^{(\alpha)}\left(\mathbf{C}^{e}: \mathbf{P}^{(\alpha)}+\mathbf{Q}^{(\alpha)} \cdot \boldsymbol{\tau}-\boldsymbol{\tau} \cdot \mathbf{Q}^{(\alpha)}\right)=\mathbf{C}^{e}: \mathbf{D}-\sum_{\alpha=1}^{m} \dot{\gamma}^{(\alpha)} \boldsymbol{\lambda}^{(\alpha)}
\end{aligned}
$$

Substituting Eq. (48) into above expression, the resulting constitutive law is expressed as

$$
\stackrel{\nabla}{\tau}=\mathbf{C}^{\text {epd }}: \mathbf{D}=\left(\mathbf{C}^{e}-\sum_{\alpha=1}^{m} \sum_{\beta=1}^{m}\left(g_{\alpha \beta}\right)^{-1} \lambda^{(\alpha)} \otimes \lambda^{(\beta)}\right): \mathbf{D}
$$

where the sum runs over the active slip components, $\mathbf{C}^{\text {epd }}$ is the elasto-plastic damage stiffness tensor, and $\mathbf{C}^{e}$ is the elastodamage stiffness tensor with components determined by Eq. (29).

To sum up, the spring-bundle and cubage components describe the elasto-damage constitutive relation and bear the stress together. Under the same stress condition, the slip components produce corresponding plastic deformation. Therefore, the plastic deformation is an attached one without influence on stress state. The coupling between plasticity and damage is excluded in above derivations. The model assembling with these three kinds of components describes the elasto-plastic damage constitutive relation under finite deformation and is called component assembling model. Different from traditional continuum models, the basic research element of this model is component rather than representative volume element, and the mechanical property of materials is described on components rather than on the representative volume element for expressing the strain-stress relations. The mechanical behavior of materials is obtained by assembling the responses of these three kinds of components.

\section{Yield surface of the proposed model}

In this section, the proposed model is used to illustrate the evolution of yield surfaces. Theoretically, the proposed model can be applied in any six-dimensional stress space, while most experimental yield surfaces found in the literature were 
determined in two-dimensional case. For the sake of clarity in discussion and verification of the proposed model, we shall restrict it in the axial-torsional stress space.

The number of slip systems in single crystal is determinate and invariant. However, the number of slip components is variant, depending on the accuracy. In the present paper, we use 30 independent slip components scatter in the first and second quadrants as shown in Fig. 2. The slip components can slip along the positive and negative directions.

The yield definition used in the present model is the absolutely proportional limit. When the resolved shear stress of a slip component reaches its critical value, this slip component actives, and then yield occurs. For the $\alpha$ slip component, the yield function can be expressed as

$$
f^{(\alpha)}=\tau^{(\alpha)}-\tau_{ \pm c r}^{(\alpha)}=\tau: \mathbf{P}^{(\alpha)}-\tau_{ \pm c r}^{(\alpha)}=0
$$

In the axial-torsional stress space, Eq. (51) can be rewritten as

$$
\tau_{11} P_{11}^{(\alpha)}+2 \tau_{12} P_{12}^{(\alpha)}=\tau_{ \pm c r}^{(\alpha)}
$$

It should be noted that yield surfaces are usually determined in Cauchy stress space. For most metals with finite plastic deformation, the elastic strain is much smaller relative to the total strain, and Cauchy stress is close to Kirchhoff stress. Eq. (52) can be approximately expressed by Cauchy stress as

$$
\sigma_{11} P_{11}^{(\alpha)}+2 \sigma_{12} P_{12}^{(\alpha)}=\tau_{ \pm c r}^{(\alpha)}
$$

The derivation of yield surface from Eq. (53) is shown in Fig. 3. It can be seen from Fig. 3 that the two state variables (the two critical resolved shear stresses, $\tau_{-c r}^{(\alpha)}$ and $\tau_{+c r}^{(\alpha)}$ ) on slip component $\alpha$ determine two critical lines. Then, running over all slip components, we can obtain a group of critical lines, and the yield surface is formed by the constraint that it is tangent to

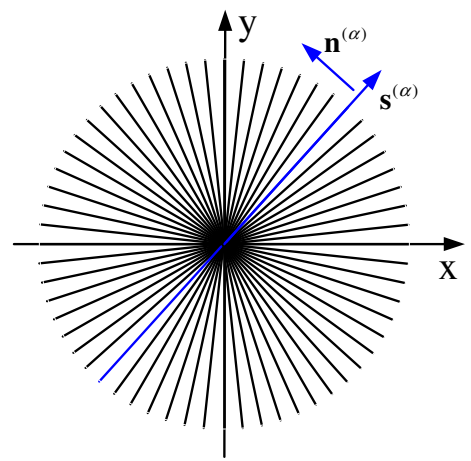

Fig. 2. The planar discrete slip directions and normal directions of slip components.

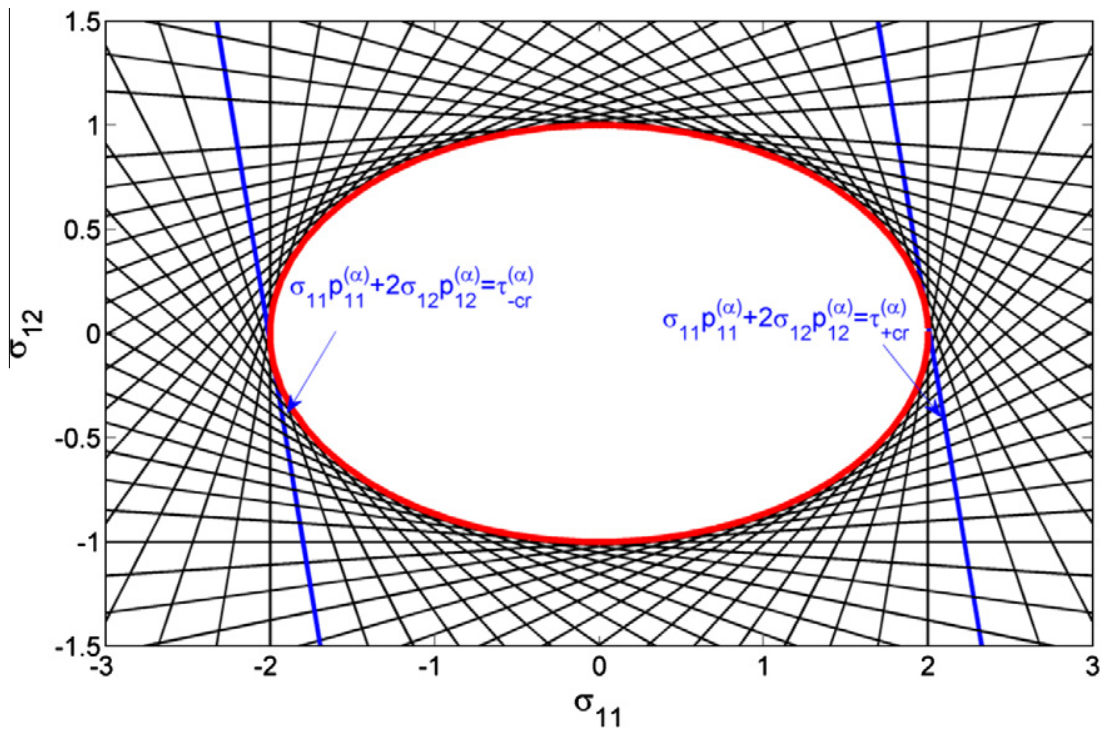

Fig. 3. A typical example of yield surface predicted by the proposed model. 


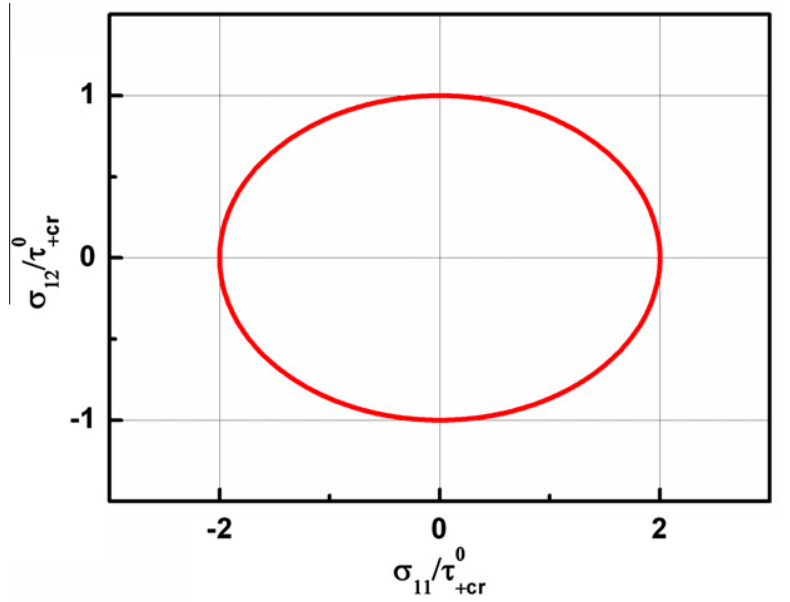

Fig. 4. The initial yield surface predicted by present model.

every critical line. Therefore, if the critical resolved shear stresses of all the slip components are determined, the yield surface is then obtained.

\subsection{The initial yield surface (IYS)}

When the critical resolved shear stresses on every slip component are equal to the initial values $\tau_{+c r}^{0}$ or $\tau_{-c r}^{0}\left(\tau_{-c r}^{0}=-\tau_{+c r}^{0}\right)$, the initial yield surface is obtained and shown in Fig. 4. Obviously, it represents the Tresca yield surface. The initial critical shear stress $\tau_{+c r}^{0}$ can be determined by the axial yield stress $\sigma_{s}$ or the shear yield stress $\tau_{s}$

$$
\tau_{+c r}^{0}=\frac{\sigma_{s}}{2} \text { or } \tau_{+c r}^{0}=\tau_{s}
$$

\subsection{The subsequent yield surface (SYS)}

There are many factors that affect the evolution of critical resolved shear stresses $\tau_{ \pm c r}^{(\alpha)}$. In the present paper, we mainly consider hardening and Bauschinger effect of the slip components. For an active slip component, the active critical resolved shear stress changes due to the self hardening, while for a non-active slip component, the active critical resolved shear stress changes due to the latent hardening. For all slip components, the passive critical resolved shear stresses change due to Bauschinger effect.

In this work, the geometric approach is used to describe the subsequent yield surface with the help of a mapping technique that divides the yield surface into the forward and rear parts. The forward part is determined by the active critical resolved shear stresses which change due to hardening, while the rear part is determined by the passive critical resolved shear stresses which change according to the Bauschinger effect. Fig. 5 is a representation of the subsequent yield surface in axial-torsional stress space based on present model.

\subsubsection{Hardening rule}

The hardening matrix $h_{\alpha \beta}$ has a crucial role in physical phenomena like cross hardening and distortion of yield surfaces. The investigation of work hardening rules has attracted the interest of many researchers. There are many kinds of hardening rules (Khan and Cheng, 1996). Considering experimental observation and theoretical study, the hardening rule in the present paper includes isotropic and kinematic hardening, and it is defined as

$$
h_{\alpha \beta}=h_{i} \operatorname{sign}\left(\mathbf{P}^{(\alpha)}: \mathbf{P}^{(\beta)}\right)+h_{k l}^{(\alpha, \beta)} \mathbf{P}^{(\alpha)}: \mathbf{P}^{(\beta)}+\left(h_{k s}^{(\alpha)}-h_{k l}^{(\alpha, \beta)}\right) \delta_{\alpha \beta} \mathbf{P}^{(\alpha)}: \mathbf{P}^{(\beta)}
$$

where $h_{i}^{(\alpha, \beta)}=h_{i} \operatorname{sign}\left(\mathbf{P}^{(\alpha)}: \mathbf{P}^{(\beta)}\right)$ represents isotropic hardening, $h_{k}^{(\alpha, \beta)}=h_{k l}^{(\alpha, \beta)} \mathbf{P}^{(\alpha)}: \mathbf{P}^{(\beta)}+\left(h_{k s}^{(\alpha)}-h_{k l}^{(\alpha, \beta)}\right) \delta_{\alpha \beta} \mathbf{P}^{(\alpha)}: \mathbf{P}^{(\beta)}$ represents kinematic hardening, where $h_{k s}^{(\alpha)}$ and $h_{k l}^{(\alpha, \beta)}$ denote self and latent hardening parameters of the kinematic hardening part respectively, and $\delta_{\alpha \beta}$ is the Kronecker delta.

The above hardening rule includes self and latent hardening. The self hardening modulus is

$$
h_{\alpha \alpha}=h_{i}+h_{k s}^{(\alpha)} \mathbf{P}^{(\alpha)}: \mathbf{P}^{(\alpha)}
$$

The latent hardening modulus is

$$
h_{\alpha \beta}=h_{i} \operatorname{sign}\left(\mathbf{P}^{(\alpha)}: \mathbf{P}^{(\beta)}\right)+h_{k l}^{(\alpha, \beta)} \mathbf{P}^{(\alpha)}: \mathbf{P}^{(\beta)} \quad(\alpha \neq \beta)
$$




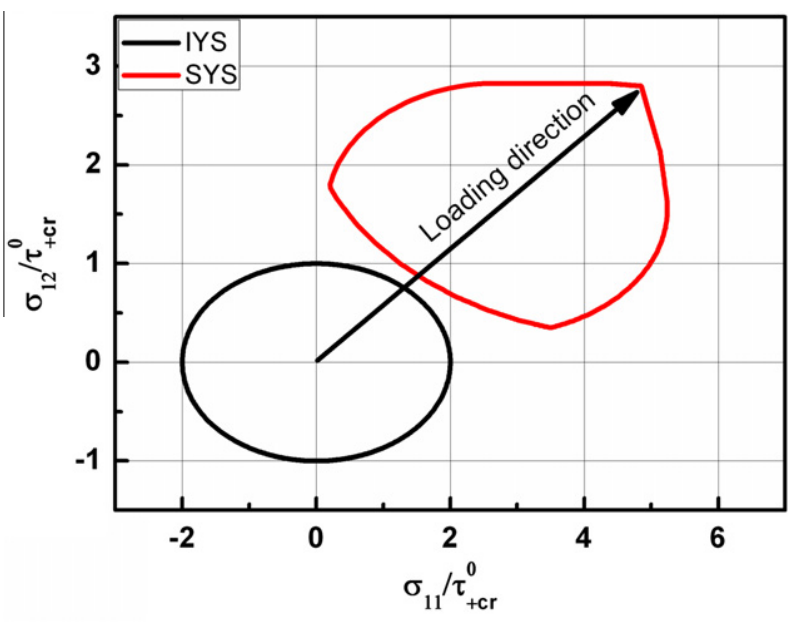

Fig. 5. Representation of the subsequent yield surface in axial-torsional stress space.

Similar to the exponential law of macroscopic strain-stress curve, the isotropic hardening parameter $h_{i}$ follows an exponential law expressed as

$$
h_{i}=2 c_{i} m_{i}\left(\left|\varepsilon^{p}\right|+\varepsilon_{0}\right)^{m_{i}-1}
$$

where $\varepsilon^{p}$ is the equivalent plastic strain, $\varepsilon^{p}=\sqrt{\left(E_{11}^{p}\right)^{2}+\frac{4}{3}\left(E_{12}^{p}\right)^{2}}$, where $\mathbf{E}^{p}$ is the Green strain. $c_{i}$ and $m_{i}$ are the isotropic hardening parameters of the model, and $\varepsilon_{0}$ is related to the initial critical resolved shear stress by

$$
\varepsilon_{0}=\left(\frac{\tau_{+c r}^{0}}{c_{i}}\right)^{\frac{1}{m_{i}}}
$$

Similarly, the self hardening parameter $h_{k s}^{(\alpha)}$ is a function of shearing deformation of slip component $\alpha$, and it is expressed as

$$
h_{k s}^{(\alpha)}=2 c_{k} m_{k}\left(\left|\gamma^{(\alpha)}\right|+\gamma_{0}\right)^{m_{k}-1}
$$

where $c_{k}$ and $m_{k}$ are the kinematic hardening parameters of the model, and $\gamma_{0}$ can be determined by

$$
\gamma_{0}=\left(\frac{\tau_{+c r}^{0}}{c_{k}}\right)^{\frac{1}{m_{k}}}
$$

Considering the symmetry of the hardening matrix and that the earlier active slip components affect the later ones, the latent hardening parameter $h_{k l}^{(\alpha, \beta)}$ can be expressed as

$$
h_{k l}^{(\alpha, \beta)}=q \min \left(h_{k s}^{(\alpha)}, h_{k s}^{(\beta)}\right)
$$

where $q$ is the latent hardening parameter.

In order to illustrate the physical meaning of the model parameters, the influence of the latent hardening, Bauschinger effect, the isotropic hardening and self hardening of kinematic hardening part is investigated in terms of subsequent yield surfaces.

\subsubsection{The latent hardening}

The influence of latent hardening parameter $q$ on subsequent yield surfaces under uniaxial tension is shown in Fig. 6. In this illustration, the isotropic hardening is not included, and $b=1$. It can be seen that the latent hardening parameter $q$ mainly affects the forward part of subsequent yield surfaces. When $q=0$, there is no latent hardening in the kinematic hardening part, and the subsequent yield surface exhibits a sharp corner with highest extent. $q=1$ corresponds to the maximum latent hardening, and classic kinematic hardening is obtained. In general, $0 \leqslant q \leqslant 1$, and the subsequent yield surfaces can be described to have a sharp front. Therefore, the model can describe the front part of the subsequent yield surface as a corner with varying degrees according to the different values of latent hardening parameter $q$.

The sharp front can be explained in terms of the critical lines determined by the active critical resolved stresses of active slip components. When $q<1$, there is usually several active slip components. For an active slip component $\alpha$, there is 


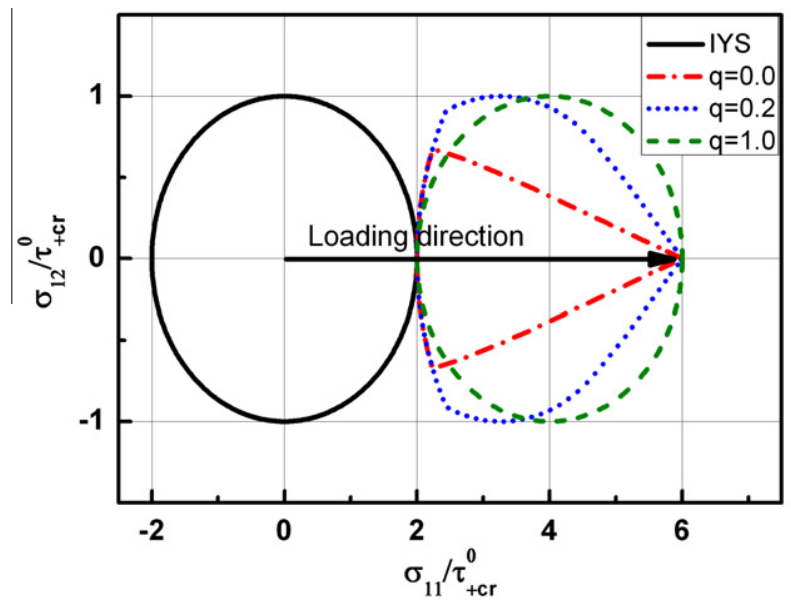

Fig. 6. The effect of latent hardening parameter $q$ on subsequent yield surfaces under uniaxial tension

$$
\begin{cases}\tau_{+c r}^{(\alpha)}=\tau^{(\alpha)}=J \boldsymbol{\sigma}_{0}: \mathbf{P}, & \dot{\tau}^{(\alpha)}>0 \\ \tau_{-c r}^{(\alpha)}=\tau^{(\alpha)}=J \boldsymbol{\sigma}_{0}: \mathbf{P}, & \dot{\tau}^{(\alpha)}<0\end{cases}
$$

where $\boldsymbol{\sigma}_{0}$ denotes the stress at the loading point. Then, the active critical line of the active slip component $\alpha$ becomes

$$
\sigma_{11} P_{11}^{(\alpha)}+2 \sigma_{12} P_{12}^{(\alpha)}=\sigma_{11}^{0} P_{11}^{(\alpha)}+2 \sigma_{12}^{0} P_{12}^{(\alpha)}
$$

That is to say, the critical lines determined by the active slip components all intersect at the loading point $\left(\sigma_{11}^{0}, \sigma_{12}^{0}\right)$, as seen in Fig. 7. Then, the subsequent yield surface determined by the critical lines will form a sharp corner at the loading point. The smaller the value of $q$ is, the larger the number of active slip components is, and the sharper the front of subsequent yield surface becomes.

\subsubsection{Bauschinger effect}

Bauschinger effect refers to that an increase of the tensile yield strength may result in a decrease of the compressive yield strength. In the present model, Bauschinger effect is defined on the slip component. It refers to that an increase of active critical resolved shear stress may result in a decrease of passive critical resolved shear stress.

Since the hardening rule in the present model is composed of two parts, the active critical resolved shear stress rate can also be divided into two parts as

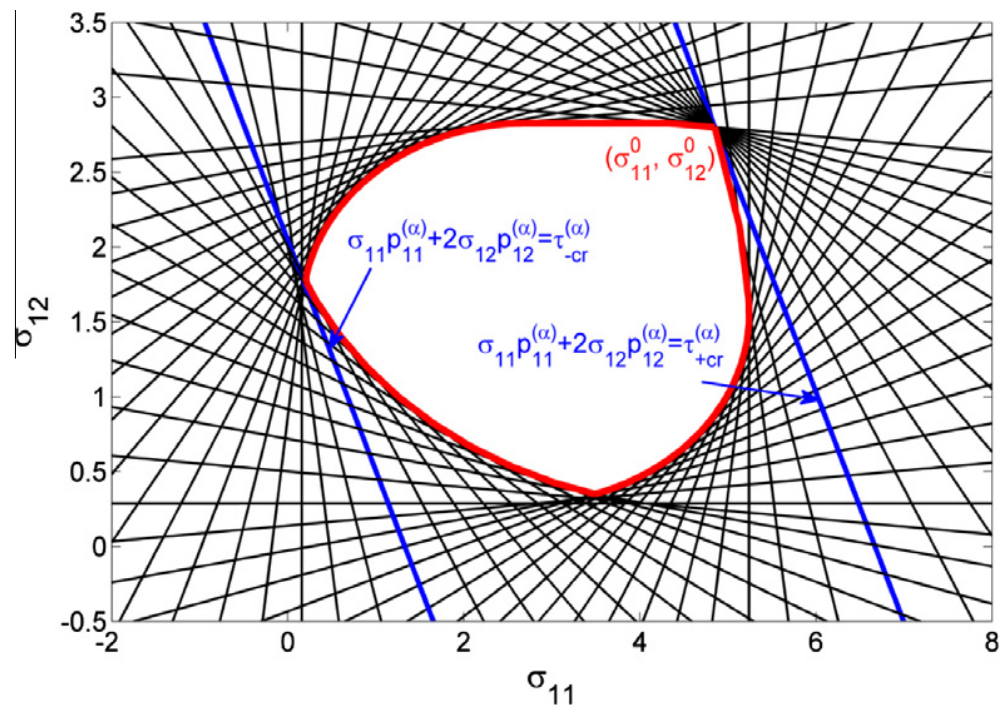

Fig. 7. An explanation of sharp front and blunt rear of subsequent yield surface in terms of critical lines. 


$$
\begin{cases}\dot{\tau}_{+c r}^{(\alpha)}=\sum_{\beta=1}^{m}\left(h_{i}^{(\alpha, \beta)}+h_{k}^{(\alpha, \beta)}\right) \dot{\gamma}^{(\beta)}=\dot{\tau}_{+c r(i)}^{(\alpha)}+\dot{\tau}_{+c r(k)}^{(\alpha)}, & \sum_{\beta=1}^{m} h_{\alpha \beta} \dot{\gamma}^{(\beta)}>0 \\ \dot{\tau}_{-c r}^{(\alpha)}=\sum_{\beta=1}^{m}\left(h_{i}^{(\alpha, \beta)}+h_{k}^{(\alpha, \beta)}\right) \dot{\gamma}^{(\beta)}=\dot{\tau}_{-c r(i)}^{(\alpha)}+\dot{\tau}_{-c r(k)}^{(\alpha)}, & \sum_{\beta=1}^{m} h_{\alpha \beta} \dot{\gamma}^{(\beta)}<0\end{cases}
$$

where $\dot{\tau}_{ \pm c r(i)}^{(\alpha)}=\sum_{\beta=1}^{m} h_{i}^{(\alpha, \beta)} \dot{\gamma}^{(\beta)}$ and $\dot{\tau}_{ \pm c r(k)}^{(\alpha)}=\sum_{\beta=1}^{m} h_{k}^{(\alpha, \beta)} \dot{\gamma}^{(\beta)}$ represent critical resolved shear stress rates originating from isotropic and kinematic hardening part respectively. For slip component $\alpha$, when $\sum_{\beta=1}^{m} h_{\alpha \beta} \dot{\gamma}^{(\beta)}>0$, the positive critical resolved shear stress $\tau_{+c r}^{(\alpha)}$ is the active one, and the negative critical resolved shear stress $\tau_{-c r}^{(\alpha)}$ is the passive one. When $\sum_{\beta=1}^{m} h_{\alpha \beta} \dot{\gamma}^{(\beta)}<0$, the negative critical resolved shear stress becomes the active one, and the positive critical resolved shear stress corresponds to the passive one.

In the present model, Bauschinger effect is defined on the kinematic hardening part of the slip component as the passive critical resolved shear stress rate of the kinematic part divided by the active one

$$
\begin{cases}b=\frac{i_{-c r(k)}^{(\alpha)}}{\dot{\tau}_{+c r(k)}^{(\alpha)}}, & \sum_{\beta=1}^{m} h_{\alpha \beta} \dot{\gamma}^{(\beta)}>0 \\ b=\frac{\dot{\tau}_{+c r(k)}^{(\alpha)}}{\dot{i}_{-c r(k)}^{(\alpha)}}, & \sum_{\beta=1}^{m} h_{\alpha \beta} \dot{\gamma}^{(\beta)}<0\end{cases}
$$

When $\sum_{\beta=1}^{m} h_{\alpha \beta} \dot{\gamma}^{(\beta)}>0$, the positive critical resolved shear stress $\tau_{+c r}^{(\alpha)}$ of $\alpha$ slip component changes due to hardening, then the Bauschinger effect parameter $b$ is equal to the rate of negative critical resolved shear stress of the kinematic part divided by the rate of positive one, and vice versa.

Therefore, the passive critical resolved shear stress can be expressed as

$$
\begin{cases}\dot{\tau}_{-c r}^{(\alpha)}=\dot{\tau}_{-c r(i)}^{(\alpha)}+\dot{\tau}_{-c r(k)}^{(\alpha)}=-\dot{\tau}_{+c r(i)}^{(\alpha)}+b \dot{\tau}_{+c r(k)}^{(\alpha)}, & \sum_{\beta=1}^{m} h_{\alpha \beta} \dot{\gamma}^{(\beta)}>0 \\ \dot{\tau}_{+c r}^{(\alpha)}=\dot{\tau}_{+c r(i)}^{(\alpha)}+\dot{\tau}_{+c r(k)}^{(\alpha)}=-\dot{\tau}_{-c r(i)}^{(\alpha)}+b \dot{\tau}_{-c r(k)}^{(\alpha)}, & \sum_{\beta=1}^{m} h_{\alpha \beta} \dot{\gamma}^{(\beta)}<0\end{cases}
$$

Generally speaking, the active critical resolved shear stress changes due to hardening as Eq. (65), and the passive one changes through Bauschinger effect as Eq. (67). The critical resolved shear stresses on all slip components determine the yield surface.

Theoretically, the Bauschinger effect parameter $b$ on slip components depends on the slip shearing deformation history, and it is different for each slip component. For simplicity, $b$ is considered to be an identical constant for all slip components in this paper.

The influence of Bauschinger effect parameter $b$ on subsequent yield surfaces under uniaxial tension is shown in Fig. 8. In this illustration, isotropic hardening is also not included, and $q=0.3$. There is no Bauschinger effect when $b=0$, while $b=1$ denotes the classical kinematic hardening.

Under the Bauschinger effect, the subsequent yield surfaces have a blunt rear. This can be explained by the reason that the rear part of subsequent yield surface is determined by the passive critical resolved shear stresses of active slip components which change more than those of the inactive slip components.

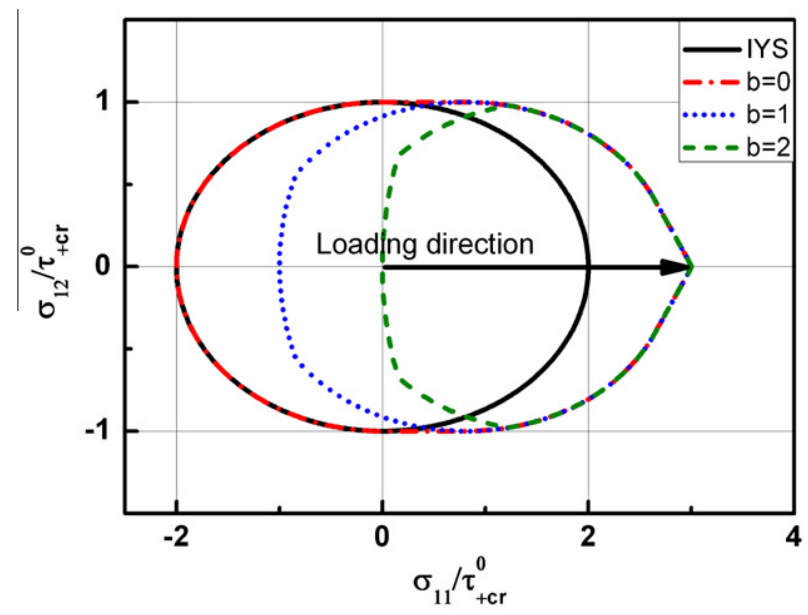

Fig. 8. The effect of Bauschinger effect parameter $b$ on the subsequent yield surfaces under uniaxial tension. 


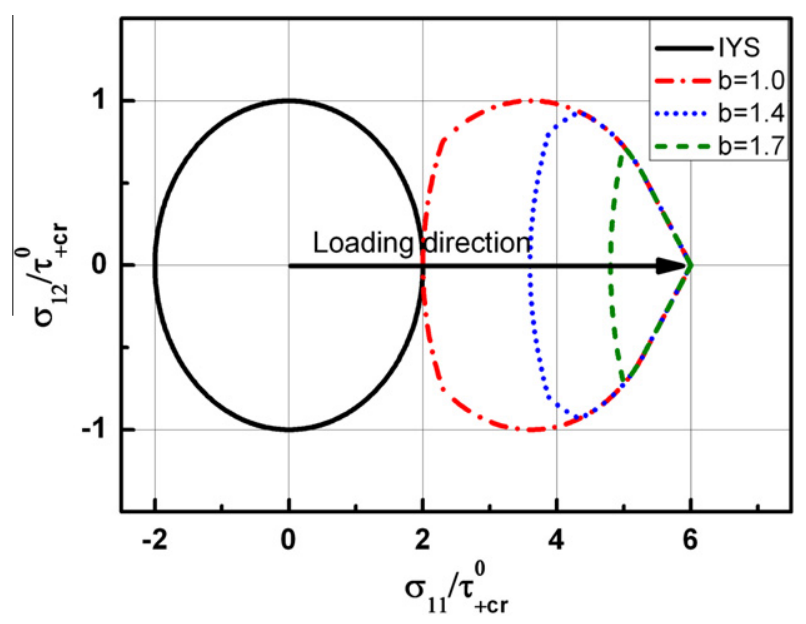

Fig. 9. Combined effect of latent hardening and Bauschinger effect on the subsequent yield surfaces under uniaxial tension ( $q=0.5$ ).

\subsubsection{Combined effect of latent hardening and Bauschinger effect}

When Bauschinger effect parameter $b$ is greater than 1 and the latent hardening parameter $q$ is smaller than 1 , the subsequent yield surfaces show slight negative cross effect in the case of finite deformation because of the combined effect of latent hardening and Bauschinger effect. When $q=0.5$, the subsequent yield surfaces under tension with different values of Bauschinger effect are shown in Fig. 9.

\subsubsection{The isotropic hardening}

Compared to kinematic hardening, the isotropic hardening is equivalent to have a constant Bauschinger effect with value -1 on slip component. That is to say, the isotropic hardening leads to that an increase of the active critical resolved shear stress may result in a same increase of passive critical resolved shear stress if only isotropic hardening is included in the hardening rule. Therefore, the isotropic hardening makes the size of subsequent yield surface increase. The subsequent yield surfaces under tension with different values of isotropic hardening are shown in Fig. 10, in which $q=0.3, b=2.0$, and $c_{i}=c_{k}=1$. As isotropic hardening takes up larger proportion of the total hardening matrix, the subsequent yield surfaces become larger and show apparent positive cross effect. The expansion of the rear part of subsequent yield surfaces is very noticeable, while the isotropic hardening has little effect on the front of the subsequent yield surfaces.

When $q=1, b=1$, the classic isotropic hardening is obtained when $h_{k}=0$, the classic kinematic hardening is obtained when $h_{i}=0$, and combined isotropic and kinematic hardening is obtained when two kinds of hardening both exist, as shown in Fig. 11.

\subsubsection{The self hardening of kinematic hardening part}

Since the strain-stress curve is determined by the hardening rule, the self hardening parameters $c_{k}$ and $m_{k}$, latent hardening parameter $q$ of the kinematic hardening part, and isotropic hardening parameters $c_{i}$ and $m_{i}$ all have influence on the

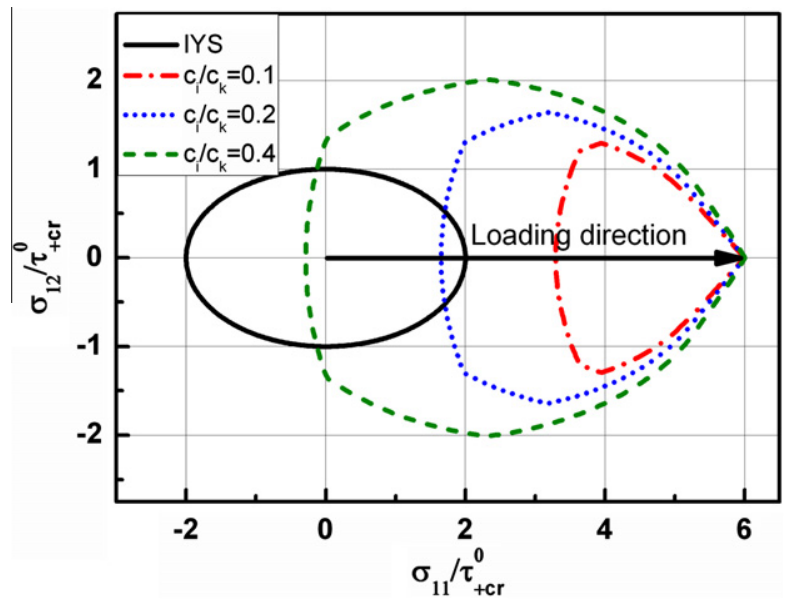

Fig. 10. The effect of isotropic hardening on the subsequent yield surfaces under uniaxial tension $\left(q=0.3, b=2.0\right.$, and $\left.m_{i}=m_{k}=1\right)$. 


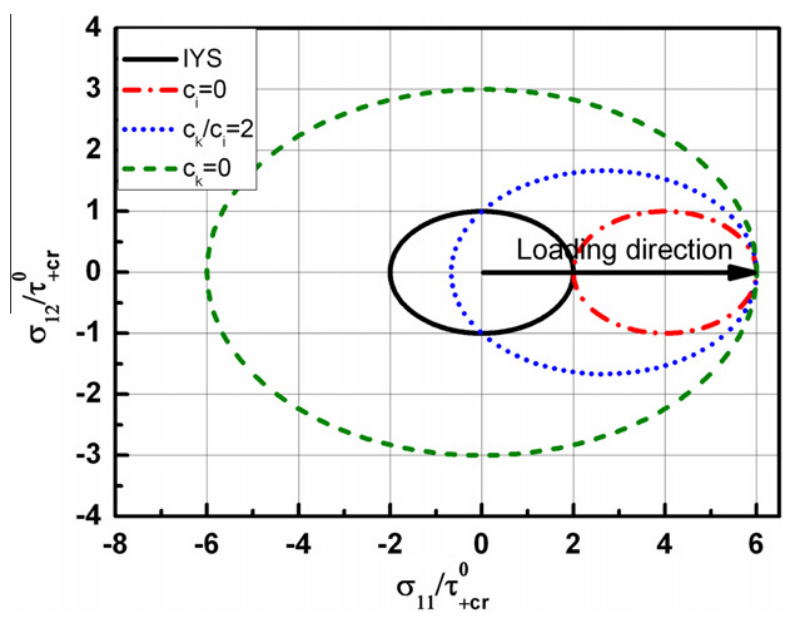

Fig. 11. The classic hardening models obtained by the present model under tension $\left(q=1, b=1\right.$, and $\left.m_{i}=m_{k}=1\right)$

strain-stress curve. Considering that the isotropic hardening makes the subsequent yield surfaces inflate, it is possible to distinguish between the isotropic hardening and kinematic hardening from their influences on the cross effect of subsequent yield surfaces. Once the isotropic hardening parameters and latent hardening parameter $q$ are determined, the self hardening parameters $c_{k}$ and $m_{k}$ can be determined by fitting the strain-stress curve.

\subsubsection{Non-proportional loading}

The calculation of non-proportional loading is an important issue in plasticity and damage mechanics. How to reflect the phenomenon that material response depends on its deformation history is difficult for many phenomenological models. In the present model, the state variables on slip components (the positive and negative critical resolved shear stresses, $\tau_{+c r}^{(\alpha)}$ and $\left.\tau_{-c r}^{(\alpha)}\right)$ are naturally different according to their different orientations. Since the subsequent yield surface is determined by the positive and negative critical resolved shear stresses on all slip components, the different critical resolved shear stresses on different slip components would create anisotropy for the material in a natural manner. Moreover, the evolution of the state variables records the loading history of material, which makes the proposed model have the potential to address the issue of complex loading. The subsequent yield surfaces under five different loading paths ((a) tension followed by torsional loading; (b) tension, unloading to the center of the yield surface followed by torsional loading; (c) combined tension-torsion proportional loading; (d) torsion followed by tensile loading; (e) torsion, unloading to the center of the yield surface followed by tensile loading) with the same loading point are shown in Fig. 12, in which classic kinematic hardening rule is used, i.e. $q=1$, $b=1$, and $h_{i}=0$. It can be seen from Fig. 12 that different subsequent yield surfaces are obtained under different loading paths.

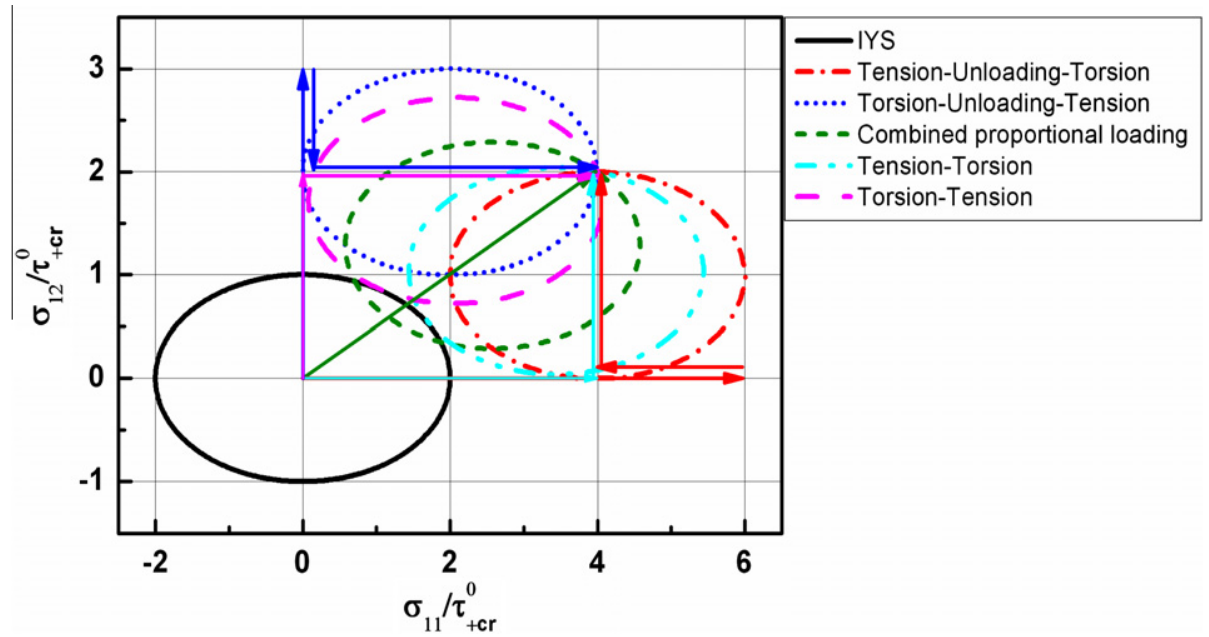

Fig. 12. The effect of loading path on the subsequent yield surfaces. 


\section{Iteration procedure}

The responses of spring-bundle and cubage components which describe the elasto-damage constitutive relation are determined by the elastic deformation gradient $\mathbf{F}^{e}$, while the responses of slip components which describe the plastic deformation behavior are controlled by the elastic deformation gradient $\mathbf{F}^{e}$ and Kirchhoff stress $\tau$. Therefore, the realization of the elasto-plastic damage constitutive relation needs iteration. On the other hand, the determination whether the slip components are active or not also needs iteration. The whole calculation procedure is presented with the condition that the nominal stress is given:

1. The nominal stress $\mathbf{S}$ at the increment $n$ is given, and the elasto-plastic damage stiffness tensor $\left(\mathbf{C}^{\text {epd }}\right)^{(n-1)}$, the elastodamage stiffness tensor $\left(\mathbf{C}^{e}\right)^{(n-1)}$ and elastic deformation gradient $\left(\mathbf{F}^{e}\right)^{(n-1)}$ at the increment $(n-1)$ are known and set as the initial iteration values of corresponding parameters at the increment $n$. The spin tensor of the elastic part $\mathbf{W}^{e}$ is set as zero;

2. Calculate Kirchhoff stress according to $\tau=\mathbf{F}^{e} \cdot \mathbf{S}$;

3. Calculate the Jaumann rate of Kirchhoff stress based on the lattice spin $\mathbf{W}^{e}$ :

$$
{\stackrel{\Delta}{\left(\mathbf{W}^{e}\right)}}=\Delta \mathbf{F}^{e} \cdot \mathbf{S}+\mathbf{F}^{e} \cdot \Delta \mathbf{S}+\tau \cdot \mathbf{W}^{e}-\mathbf{W}^{e} \cdot \tau
$$

4. Compute the elastic deformation rate according to $\mathbf{D}^{e}=\left(\mathbf{C}^{e}\right)^{-1}: \stackrel{\Delta}{\boldsymbol{\tau}}_{\left(\mathbf{W}^{e}\right)}$;

5. Compute the increment of elastic deformation gradient $\Delta \mathbf{F}^{e}=\left(D^{e}+W^{e}\right) \cdot \mathbf{F}^{e}$, and the new total elastic deformation gradient $\mathbf{F}_{e}=\Delta \mathbf{F}_{e}+\mathbf{F}_{e}^{(n-1)}$;

6. Compute the direction vector of every spring-bundle component after deformation according to $\mathbf{n}^{(s)}=\mathbf{F}^{e} \cdot \mathbf{N}^{(s)}$ and its deformation $\lambda^{(s)}=\frac{1}{2} \ln \left(\mathbf{n}^{(s)} \cdot \mathbf{n}^{(s)}\right)$. According to the response curve of the spring-bundle component, the force $f\left(\lambda^{(s)}\right)$ and stiffness $H\left(\lambda^{(s)}\right)$ of every spring-bundle component are determined;

7. Compute the deformation of the cubage component $\theta=\ln \left(\operatorname{det} \mathbf{F}^{e}\right)$, according to the response curve of cubage component, the force $p(\theta)$ and stiffness $K(\theta)$ of the cubage component are determined;

8. Compute the Kirchhoff stress according to Eq. (26) and elasto-damage stiffness tensor $\mathbf{C}^{e}$ according to Eq. (29);

9. Using Kirchhoff stress, the nominal stress is calculated by $\tilde{\mathbf{S}}=\left(\mathbf{F}^{e}\right)^{-1} \cdot \tau$;

10. A condition $\|\mathbf{S}-\tilde{\mathbf{S}}\| \leqslant \delta$ ( $\delta$ is a small value dependent on the calculation precision) comparing the computed nominal stress and the given one is used to verify the convergence of the iteration. If the condition is satisfied, proceed to the next step, otherwise, assign the value $\Delta \mathbf{S}+(\mathbf{S}-\tilde{\mathbf{S}})$ to $\Delta \mathbf{S}$. Go back to step 3 using the updated variables obtained in above iteration process;

11. Compute the slip direction and the corresponding normal of every slip component after deformation according to Eqs. (31) and (32), respectively. Compute second-order symmetric tensor $\mathbf{P}^{(\alpha)}$, antisymmetric tensor $\mathbf{Q}^{(\alpha)}$ and second-order tensor $\lambda^{(\alpha)}=\mathbf{P}^{(\alpha)}: \mathbf{C}^{e}+\mathbf{Q}^{(\alpha)} \cdot \tau-\tau \cdot \mathbf{Q}^{(\alpha)}$;

12. According to the hardening rule of the slip component, the hardening matrix $h_{\alpha \beta}$ is determined. Compute $g_{\alpha \beta}=h_{\alpha \beta}+\lambda^{(\alpha)}: \mathbf{P}^{(\beta)}$;

13. Calculate the resolved shear stress of every slip component according to Eq. (35). For every slip component, according to the relation between the resolved shear stress and the critical resolved shear stresses at the last increment step, try to determine whether it is active or not:

(a) If $\tau^{(\alpha)}>\left(\tau_{+c r}^{(\alpha)}\right)^{(n-1)}$, the slip component is regarded to be active and slip along the positive direction, the increment of critical resolved shear stress is computed by $\Delta \tilde{\tau}_{c r}^{(\alpha)}=\tau^{(\alpha)}-\left(\tau_{+c r}^{(\alpha)}\right)^{(n-1)}$;

(b) If $\tau^{(\alpha)}<\left(\tau_{-c r}^{(\alpha)}\right)^{(n-1)}$, the slip component is regarded to be active and slip along the negative direction, the increment of critical resolved shear stress is computed by $\Delta \tilde{\tau}_{c r}^{(\alpha)}=\tau^{(\alpha)}-\left(\tau_{-c r}^{(\alpha)}\right)^{(n-1)}$;

(c) Otherwise, the slip component is regarded to be inactive, and $\Delta \tilde{\tau}_{c r}^{(\alpha)}=0$;

14. According to the hardening matrix $h_{\alpha \beta}$ and the trial value of increment of critical resolved shear stress $\Delta \tilde{\tau}_{c r}^{(\alpha)}$, the shearing deformation rate of an active slip component $\alpha$ is computed by $\Delta \tilde{\gamma}^{(\alpha)}=\sum_{\beta=1}^{n}\left(h_{\alpha \beta}\right)^{-1} \Delta \tilde{\tau}_{c r}^{(\beta)}$, while $\Delta \tilde{\gamma}^{(\alpha)}=0$ for inactive slip component;

15. Compute the trial values of the active critical resolved shear stresses of slip components according to the following rule

(a) If $\sum_{\beta=1}^{m} h_{\alpha \beta} \Delta \gamma^{(\beta)}>0$, then $\tilde{\tau}_{+c r}^{(\alpha)}=\left(\tau_{+c r}^{(\alpha)}\right)^{(n-1)}+\sum_{\beta=1}^{m} h_{\alpha \beta} \Delta \gamma^{(\beta)}$;
(b) If $\sum_{\beta=1}^{m} h_{\alpha \beta} \Delta \gamma^{(\beta)}<0$, then $\tilde{\tau}_{-r)}^{(\alpha)}=\left(\tau_{-r)}^{(\alpha)}\right)^{(n-1)}+\sum_{\beta=1}^{m} h_{\alpha \beta} \Delta \gamma^{(\beta)}$;

16. For every slip component, determine whether the conditions $\Delta \tilde{\tau}_{c r}^{(\alpha)} \Delta \tilde{\gamma}^{(\alpha)} \geqslant 0$ and $\tilde{\tau}_{-c r}^{(\alpha)}<\tau^{(\alpha)}<\tilde{\tau}_{+c r}^{(\alpha)}$ are satisfied. If the two conditions are both satisfied for all slip components, proceed to the next step, otherwise

(a) If the condition $\tilde{\tau}_{-c r}^{(\alpha)}<\tau^{(\alpha)}<\tilde{\tau}_{+c r}^{(\alpha)}$ is satisfied for every slip component, the slip component with the minimal absolute increment of critical resolved shear stress is regarded to be inactive. Let the increment of critical resolved shear stress of this slip component be zero, $\Delta \tilde{\tau}_{c r}^{(\alpha)}=0$, and go back to step 14;

(b) If the condition $\tilde{\tau}_{-c r}^{(\alpha)}<\tau^{(\alpha)}<\tilde{\tau}_{+c r}^{(\alpha)}$ is not satisfied for slip component $\alpha$, this slip component is regarded to be active. Let the increment of critical resolved shear stress of this slip component returns to its original value computed in step 13 , and go back to step 14 ; 
17. Compute the increments of critical resolved shear stresses which are composed of the isotropic hardening part $\Delta \tau_{ \pm c r(i)}^{(\alpha)}=\sum_{\beta=1}^{m} h_{i}^{(\alpha, \beta)} \Delta \gamma^{(\beta)}$ and the kinematic hardening part $\Delta \tau_{ \pm c r(k)}^{(\alpha)}=\sum_{\beta=1}^{m} h_{k}^{(\alpha, \beta)} \Delta \gamma^{(\beta)}$. The positive and negative critical resolved shear stresses are calculated:

(a) If $\sum_{\beta=1}^{m} h_{\alpha \beta} \Delta \gamma^{(\beta)}>0$, the positive critical resolved shear stress is calculated by $\tau_{+c r}^{(\alpha)}=\left(\tau_{+c r}^{(\alpha)}\right)^{(n-1)}+\Delta \tau_{+c r(i)}^{(\alpha)}+\Delta \tau_{+c r(k)}^{(\alpha)}$, while the negative critical resolved shear stress is calculated by $\tau_{-c r}^{(\alpha)}=\left(\tau_{-c r}^{(\alpha)}\right)^{(n-1)}-\Delta \tau_{+c r(i)}^{(\alpha)}+b \Delta \tau_{+c r(k)}^{(\alpha)}$;

(b) If $\sum_{\beta=1}^{m} h_{\alpha \beta} \Delta \gamma^{(\beta)}<0$, the negative critical resolved shear stress is calculated by $\tau_{-c r}^{(\alpha)}=\left(\tau_{-c r}^{(\alpha)}\right)^{(n-1)}+\Delta \tau_{-c r(i)}^{(\alpha)}+\Delta \tau_{-c r(k)}^{(\alpha)}$, while the positive critical resolved shear stress is calculated by $\tau_{+c r}^{(\alpha)}=\left(\tau_{+c r}^{(\alpha)}\right)^{(n-1)}-\Delta \tau_{-c r(i)}^{(\alpha)}+b \Delta \tau_{-c r(k)}^{(\alpha)}$;

18. Compute the shearing deformation of every slip component by $\gamma^{(\alpha)}=\left(\gamma^{(\alpha)}\right)^{(n-1)}+\Delta \gamma^{(\alpha)}$, plastic deformation gradient by $\mathbf{F}^{p}=\mathbf{I}+\sum_{\alpha=1}^{n} \gamma^{(\alpha)} \mathbf{S}^{(\alpha)} \otimes \mathbf{N}^{(\alpha)}$, total deformation gradient by $\mathbf{F}=\mathbf{F}^{e} \cdot \mathbf{F}^{p}$ and elasto-plastic damage stiffness tensor by $\mathbf{C}^{e p d}=\mathbf{C}^{e}-\sum_{\alpha=1}^{n} \sum_{\beta=1}^{n}\left(g_{\alpha \beta}\right)^{-1} \lambda^{(\alpha)} \otimes \boldsymbol{\lambda}^{(\beta)}$;

19. Update the state variables on spring-bundle components (maximum and minimum deformations experienced by spring-bundle components) and slip components (positive and negative critical resolved shear stresses).

20. If the strain or stress reaches to the prestrain or prestress at which the subsequent yield surface is given, according to the critical resolved shear stresses on all slip components, the subsequent yield surface is determined.

\section{Results and discussion}

In this section, numerical simulations and predictions by the present model are performed to compare with two sets of experimental data by Khan et al. (2009, 2010a), one for a very low work hardening aluminum alloy Al 6061-T6511, and another for a very high work hardening aluminum alloy annealed $1100 \mathrm{Al}$. Plane stress condition is assumed in the simulations and predictions.

The experiments were conducted on the thin-walled cylindrical hollow specimens. The subsequent yield surfaces were determined during tension, torsion, and combined tension-torsion proportional/non-proportional loading paths with yield defined by $10 \mu \varepsilon$ deviation from linearity.

\subsection{Calibration of model parameters}

The Young's and shear moduli of $\mathrm{Al} \mathrm{6061-T6511}$ and annealed $1100 \mathrm{Al}$ are $70.55 \mathrm{MPa}, 25.86 \mathrm{MPa}$ and $70.49 \mathrm{MPa}$, 25.84 MPa, respectively.

In the present model, the basic research element is the three kinds of components, and the mechanical property of material is described on components. Therefore, the calibration of model parameters is the calibration of three kinds of components actually.

\subsubsection{Calibration of spring-bundle components}

Since damage is reflected by the response functions of spring-bundle and cubage components, the variation of elastic constants can be determined by the damage defined on these two kinds of components. For simplicity, the damage of cubage component is not considered in this simulation. Therefore, the evolution of elastic constants is only influenced by the response curve of spring-bundle components. Considering that the stiffness of spring-bundle component decrease exponentially, the response of the spring-bundle component can be expressed as

$$
f=\left\{\begin{array}{cc}
\left(H_{0} \lambda_{0}+H_{0} k\left(1-e^{\left.\left.\frac{-\lambda_{+h}+\lambda_{0}}{k}\right)\right) \frac{\lambda}{\lambda_{+h}}}\right.\right. & \lambda_{+h}>\lambda_{0} \\
\left(-H_{0} \lambda_{0}-H_{0} k\left(1-e^{\frac{\lambda_{-h}+\lambda_{0}}{k}}\right)\right) \frac{\lambda}{\lambda_{-h}} & \lambda_{-h}<-\lambda_{0} \\
H_{0} \lambda & -\lambda_{0} \leqslant \lambda_{ \pm h} \leqslant \lambda_{0}
\end{array}\right.
$$

where $H_{0}$ is the initial stiffness of spring-bundle component, $\lambda_{ \pm h}$ is the deformation history of a spring-bundle component with initial value $\lambda_{0}$ or $-\lambda_{0}$, and $k$ is a parameter which determines the rate of damage development of spring-bundle component. The response curve of spring-bundle component is shown in Fig. 13.

The initial stiffness of a spring-bundle component representing the atomic bonds in a little solid angle of $\Delta \Omega$ is

$$
H_{0}=h \Delta \Omega
$$

where $h$ is the stiffness of spring-bundle component in a unit solid angle. In plane stress condition, the stiffnesses of springbundle components and cubage component are related to the initial Young's and shear moduli through the comparison between the present model and the generalized Hooke's law, and they are expressed as

$$
\left\{\begin{array}{l}
h=\frac{8}{\pi} G \\
B=\frac{3 E-8 G}{4 G-E} G
\end{array}\right.
$$

The parameter $\lambda_{0}$ is determined by the strain at which the elastic constants begin to decrease, while $k$ determines the descending rate of elastic constants. Therefore, $\lambda_{0}$ and $k$ can be determined by the evolution of Young's modulus under tension. 


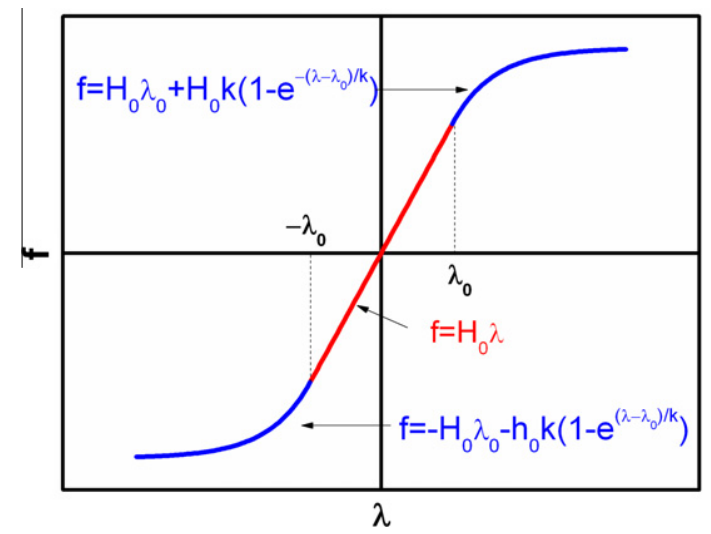

Fig. 13. The response curve of spring-bundle component.

Table 1

Parameters for simulations of the evolution of the yield surfaces and elastic constants (Al 6061-T6511).

\begin{tabular}{lllllllll}
\hline$\lambda_{0}$ & $k$ & $\tau_{+c r}^{0}(/ \mathrm{MPa})$ & $c_{i}(\mathrm{MPa})$ & $m_{i}$ & $c_{k}(\mathrm{MPa})$ & $m_{k}$ & $q$ & $b$ \\
\hline 0.0029 & 0.0017 & 60.8 & $/$ & $/$ & 143.9 & 0.08 & 0.4 & 2.15 \\
\hline
\end{tabular}

Table 2

Parameters for simulations of the evolution of the yield surfaces and elastic constants (annealed $1100 \mathrm{Al}$ ).

\begin{tabular}{lllllllll}
\hline$\lambda_{0}$ & $k$ & $\tau_{+c r}^{0}(/ \mathrm{MPa})$ & $c_{i}(\mathrm{MPa})$ & $m_{i}$ & $c_{k}(\mathrm{MPa})$ & $m_{k}$ & $q$ & $b$ \\
\hline 0.00065 & 0.0012 & 3.8 & 18.4 & 0.2 & 23.6 & 0.2 & 0.5 & 2.3 \\
\hline
\end{tabular}

The parameters of Al 6061-T6511 and annealed $1100 \mathrm{Al}$ are listed in Tables 1 and 2, respectively. Using the same parameters, the evolution of Young's modulus under other loading conditions is predicted.

\subsubsection{Calibration of cubage component}

The damage of cubage component is not considered in this application, so there is only one parameter on cubage component, the stiffness $B$ which is related to the initial elastic constants by Eq. (70).

\subsubsection{Calibration of slip components}

The parameters of slip components are determined by the strain-stress curve, the initial yield surface and subsequent yield surfaces under tension. In view of the different evolution tendencies of subsequent yield surfaces for two kinds of alloys, the hardening rules for two alloys are somewhat different. For low work hardening aluminum alloy, considering that the subsequent yield surfaces shrink in size, only kinematic hardening is included in the hardening rule, i.e. in Eq. $(55)$, $h_{i}=0$. Therefore, there are only 5 model parameters on slip components for low work hardening aluminum alloy, the initial critical resolved shear stress $\tau_{+c r}^{0}$, the latent hardening parameter $q$, the Bauschinger effect parameter $b$ and the kinematic hardening parameters $c_{k}$ and $m_{k}$. For high work hardening aluminum alloy, considering that the subsequent yield surfaces inflate, the isotropic hardening is included as well as kinematic hardening, so there are two more parameters for high work hardening aluminum alloy, the isotropic hardening parameters $c_{i}$ and $m_{i}$. The calibration process is

(1) The initial critical resolved shear stress $\tau_{+c r}^{0}$ is determined by the initial yield surface.

(2) According to the sharp extent of the front part of subsequent yield surface, the latent hardening parameter $q$ is determined.

(3) For the high work hardening aluminum alloy annealed $1100 \mathrm{Al}$, isotropic hardening parameters $c_{i}$ and $m_{i}$ are determined by the inflation of subsequent yield surfaces, while isotropic hardening is not included for the low work hardening aluminum alloy Al 6061-T6511.

(4) For the low work hardening aluminum alloy Al 6061-T6511, according to the translation of rear part relative to that of forward part, the Bauschinger effect parameter $b$ is determined. For the high work hardening aluminum alloy annealed $1100 \mathrm{Al}$, the Bauschinger effect parameter $b$ is also determined according to the translation of rear part. However, unlike Al 6061-T6511, the Bauschinger effect parameter of annealed $1100 \mathrm{Al}$ needs to consider the isotropic hardening which also influences the translation of rear part. 


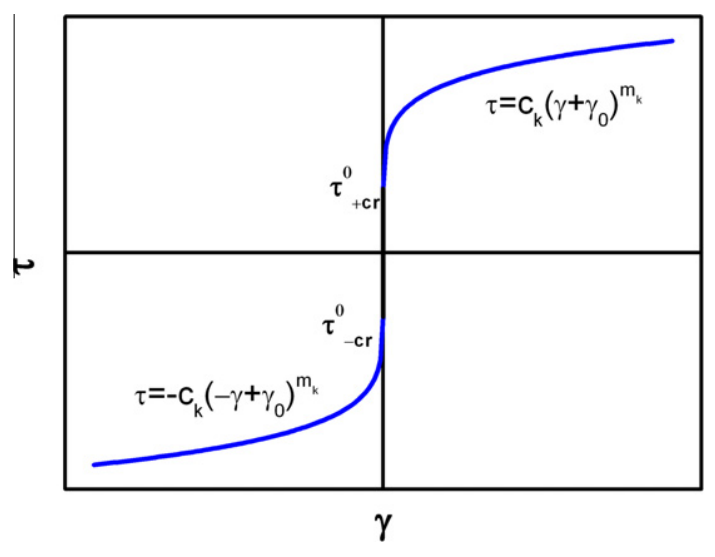

Fig. 14. The response curve of a continuously active slip component $\left(q=0, h_{i}=0\right)$.

(5) The self hardening parameters of the kinematic part, $c_{k}$ and $m_{k}$, are determined by fitting the strain-stress curve.

In fact, the competition between isotropic hardening and kinematic hardening determines the size of subsequent yield surfaces. Moreover, these two kinds of hardening both influence the strain-stress curve. Therefore, for annealed $1100 \mathrm{Al}$, the calibration of isotropic hardening and kinematic hardening parameters needs to take both the cross effect of subsequent yield surfaces and the strain-stress curve into account.

All model parameters of Al 6061-T6511 and annealed $1100 \mathrm{Al}$ are listed in Tables 1 and 2, respectively.

If there is only one slip component active in the whole plastic deformation process, or $q=0$, and that the isotropic hardening is not included, the response curve of this continuously active slip component can be seen in Fig. 14. For real materials, the response curves of slip components are different from one to another due to the influence of latent hardening and activation state.

Using these parameters, the evolution of subsequent yield surfaces under torsion and combined tension-torsion proportional loading for $\mathrm{Al}$ 6061-T6511 and annealed $1100 \mathrm{Al}$ and non-proportional loading for annealed $1100 \mathrm{Al}$ are predicted by the present model.

\subsection{The initial yield surface}

The results of the initial yield surfaces of $\mathrm{Al} \mathrm{6061-T6511} \mathrm{and} \mathrm{annealed} 1100 \mathrm{Al}$ are shown in Fig. 15(a) and (b), respectively. The theory which leads to the Tresca initial yield surface is compared with the experimental data. Although the von-Mises yield surface is closer to the experimental results than Tresca yield surface, the result predicted by the present model is also very good when we properly choose the initial resolved shear stress of slip component determined by the macro yield stress.

\subsection{The subsequent yield surfaces of proportional loading}

\subsubsection{The tensile case}

The subsequent yield surfaces of Al6061-T6511 under tensile loading are shown at 2\%, $4 \%$ and $6 \%$ true tensile strains in Fig. 16(a), and those of annealed $1100 \mathrm{Al}$ are shown at 2\%, $8 \%$ and $16 \%$ true tensile strains in Fig. 16(b). Comparisons of simulations and experimental results exhibit satisfactory agreement.

Prestrain loading is applied in all loading cases. According to the prestrain of the subsequent yield surface, the stress is obtained through the stress-strain relation, and this stress corresponds to the stress point at the front of the subsequent yield surface.

\subsubsection{The torsional case}

The subsequent yield surfaces of Al6061-T6511 under torsional loading predicted by the present model using parameters listed in Table 1 are shown at 4\%,8\% and 16\% shear strains and compared with experimental results in Fig. 17(a), and those of annealed $1100 \mathrm{Al}$ using parameters listed in Table 2 are shown at 4\%,10\% and 20\% shear strains and compared with experimental results in Fig. 17(b).

\subsubsection{The combined tension-torsional case}

The subsequent yield surfaces of Al6061-T6511 under combined tension-torsional loading predicted by the present model using parameters listed in Table 1 are shown at $2 \%, 4 \%$ and $8 \%$ equivalent von-Mises strains and compared with experi- 


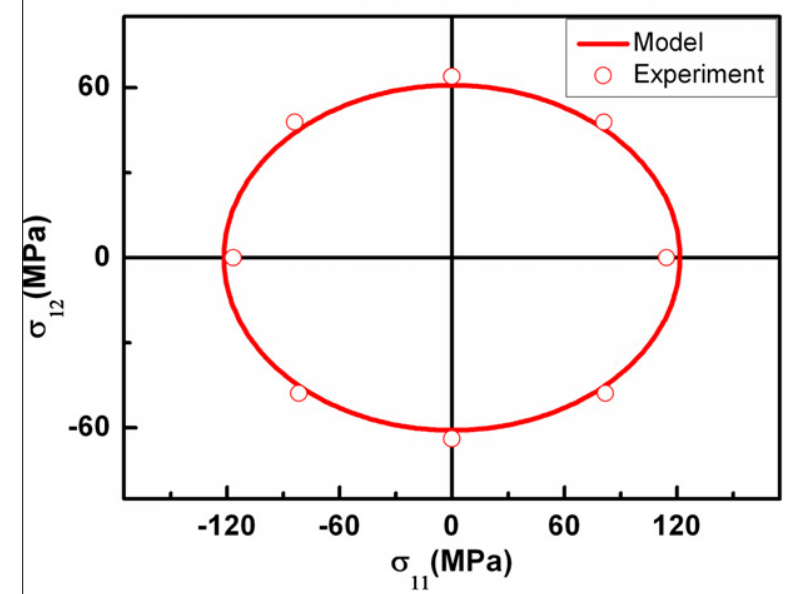

(a) $\mathrm{Al}$ 6061-T6511

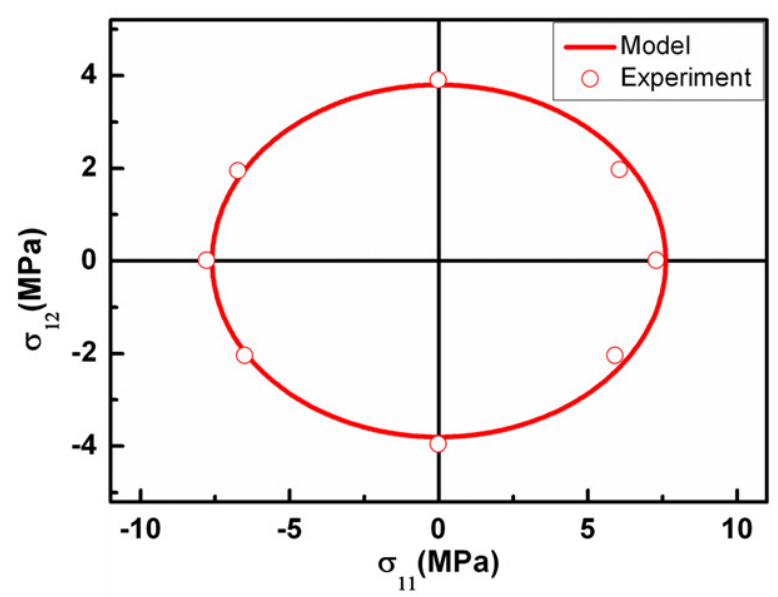

(b) annealed $1100 \mathrm{Al}$

Fig. 15. Comparisons of the initial yield surfaces between the simulations of the model and experimental data.

mental results in Fig. 18(a), and those of annealed $1100 \mathrm{Al}$ using parameters listed in Table 2 are shown at 2\%, 8\% and 16\% von-Mises strains and compared with experimental results in Fig. 18(b).

It can be seen from Fig. 16-18 that the model can characterize the evolution of subsequent yield surfaces of Al 6061T6511 and annealed $1100 \mathrm{Al}$ which showed a distinct "nose" in the loading direction for all three proportional loading paths. This can be explained by the reason that the forward part of subsequent yield surface exhibits a sharp corner because of latent hardening and the rear part forms a blunt region because of Bauschinger effect, which makes the subsequent yield surfaces exhibit a "nose". Similar nose-shaped yield surfaces are obtained by Ortiz and Popov (1983), Voyiadjis and Foroozesh (1990), Chiang et al. (2002), Yeh and Lin (2006a) and Feigenbaum and Dafalias (2007).

From Figs. 16(a)-18(a), it can be seen that the subsequent yield surfaces of Al 6061-T6511 exhibit negative cross effect for all three proportional loading paths. The shrinkage in size is because of the combined effect of latent hardening and Bauschinger effect as explained in Section 3.3.4. Unlike Al 6061-T6511, the size of subsequent yield surfaces of annealed $1100 \mathrm{Al}$ increases, and positive cross effect is observed with plastic deformation, as shown in Figs. 16(b)-18(b). This is because of the isotropic hardening of annealed $1100 \mathrm{Al}$.

\subsubsection{Discussion of the evolution rule of yield surface under proportional loading}

For any stress state on a proportional loading path, it is possible to transform it to a stress state in the principal stress space. Further, if the hydrostatic pressure is assumed to not influence the plastic deformation behavior, any proportional loading can be equivalent to a tensile case.

For a proportional loading path with loading point $\left(\sigma_{0}, \tau_{0}\right)$, its two principal stresses are

$$
\sigma_{1}=\frac{\sigma_{0}}{2}+\sqrt{\frac{\sigma_{0}^{2}}{4}+\tau_{0}^{2}}, \quad \sigma_{2}=\frac{\sigma_{0}}{2}-\sqrt{\frac{\sigma_{0}^{2}}{4}+\tau_{0}^{2}}
$$




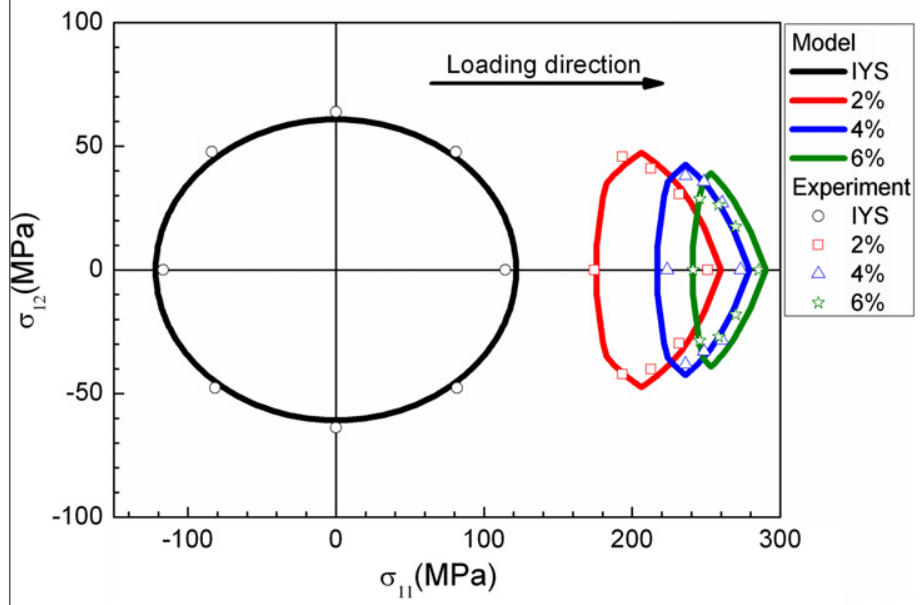

(a) $\mathrm{Al} 6061-\mathrm{T} 6511$

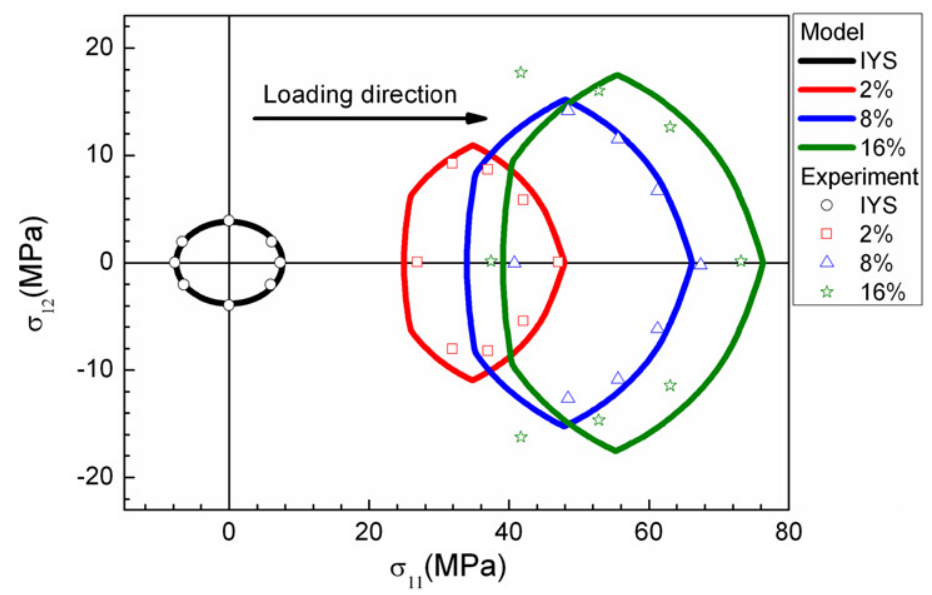

(b) annealed $1100 \mathrm{Al}$

Fig. 16. Comparison of the subsequent yield surfaces after tensile loading between the simulation of the model and experimental data.

Since hydrostatic pressure is not considered in this illustration, the above stress state can be transformed to a tensile case by subtracting the hydrostatic pressure with value $\sigma_{2}$, and the equivalent tensile stress value becomes to

$$
\sigma_{1}^{\prime}=2 \sqrt{\frac{\sigma_{0}^{2}}{4}+\tau_{0}^{2}}
$$

If the two principal directions are set as the new coordinate axes, the stress point $\left(\sigma_{11}, \sigma_{12}\right)$ on the original subsequent yield surface with preloading point $\left(\sigma_{0}, \tau_{0}\right)$ can be transformed to

$$
\left\{\begin{array}{l}
\sigma_{11}^{\prime}=\sigma_{11} \cos ^{2} \theta+\sigma_{12} \sin 2 \theta \\
\sigma_{12}^{\prime}=-\frac{1}{2} \sigma_{11} \sin 2 \theta+\sigma_{12} \cos 2 \theta \\
\sigma_{22}^{\prime}=\sigma_{11} \sin ^{2} \theta-\sigma_{12} \sin 2 \theta
\end{array}\right.
$$

where $\theta$ is the angle of the first principal direction with respect to the original $x$ axis.

Similarly, a hydrostatic pressure with value $\sigma_{22}^{\prime}$ is subtracted from the above stress state, and the new subsequent yield surface is formed by the new yield point

$$
\left\{\begin{array}{l}
\tilde{\sigma}_{11}=\sigma_{11} \cos 2 \theta+2 \sigma_{12} \sin 2 \theta \\
\tilde{\sigma}_{12}=-\frac{1}{2} \sigma_{11} \sin 2 \theta+\sigma_{12} \cos 2 \theta
\end{array}\right.
$$

In the present model, for slip component with slip direction $\alpha$, its corresponding second-order symmetric tensor $\mathbf{P}^{(\alpha)}$ has its initial components 


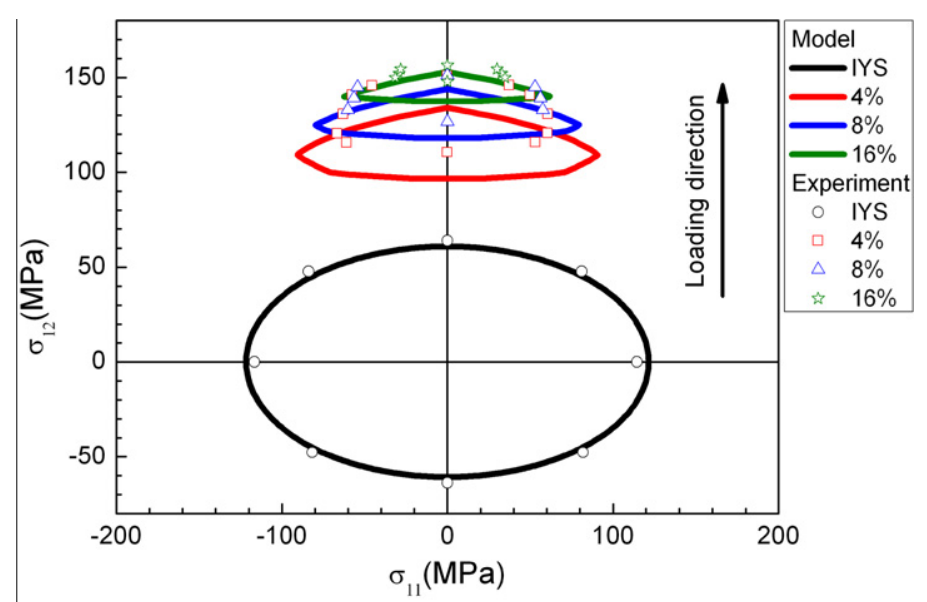

(a) Al 6061-T6511

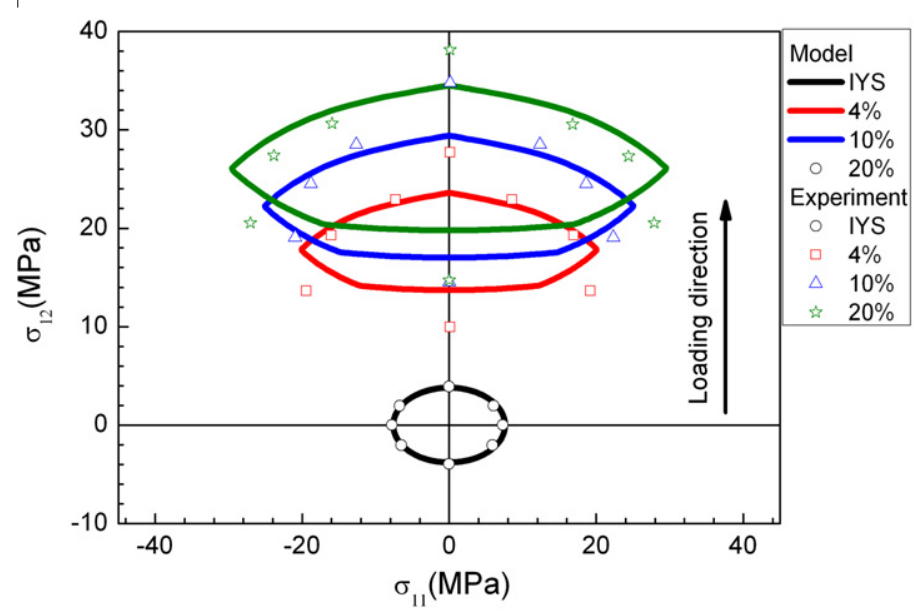

(b) annealed $1100 \mathrm{Al}$

Fig. 17. Comparison of the subsequent yield surfaces after torsional loading between the prediction of the model and experimental data.

$$
P_{11}=-\frac{1}{2} \sin 2 \alpha, \quad P_{22}=\frac{1}{2} \sin 2 \alpha
$$

If elastic deformation is not considered in the deformation of slip components, i.e. the tensor $\mathbf{P}^{(\alpha)}$ does not change, the resolved shear stress on each slip component is zero according to Eq. (35) under hydrostatic pressure. Therefore, the assumption that hydrostatic pressure doesn't influence the plastic deformation behavior holds in the present model, and any proportional loading can be transformed to an equivalent tensile case. Further, the subsequent yield surface under any proportional loading can be transformed to that under an equivalent tensile loading, and the transformed subsequent yield surface coincides with that obtained directly by tensile loading.

For three proportional loading paths in the experiment, it is possible to transform the subsequent yield surfaces to the equivalent tensile case according to the Eq. (74), the comparison results of two kinds of materials are shown in Fig. 19(a) and (b), respectively.

The difference of the subsequent yield surfaces under three proportional loading paths can be attributed to the anisotropy of materials, the different property of specimens, the deviation from the assumption that hydrostatic pressure does not influence the plastic deformation behavior for real materials, and the inevitable errors of experiments.

In the present model, the subsequent yield surfaces under three proportional loading paths coincide if they are transformed to the equivalent tensile case, while those in the experiment do not coincide. Therefore, if the subsequent yield surfaces under tensile case agree well with experimental results, the differences under other proportional loading paths between the prediction and experimental results are related to the differences between the transformed subsequent yield surfaces and those obtained directly by tensile loading. For example, the size of subsequent yield surfaces under combined tension-torsional loading in prediction results is larger than that in experiment, and it can be attributed to that the size of the transformed subsequent yield surfaces of combined tension-torsional loading case is much smaller than those obtained directly by tensile loading in experiment. 


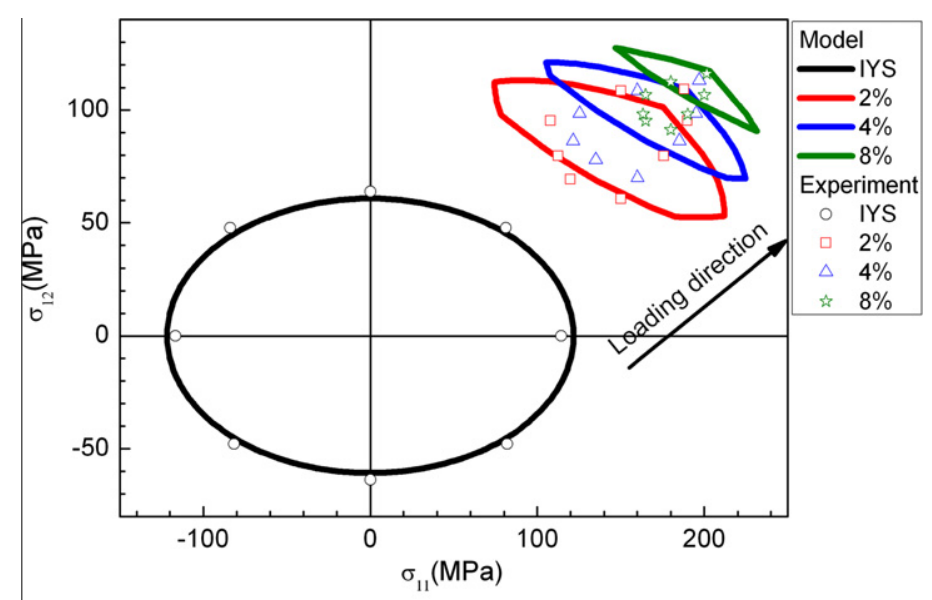

(a) $\mathrm{Al} 6061-\mathrm{T} 6511$

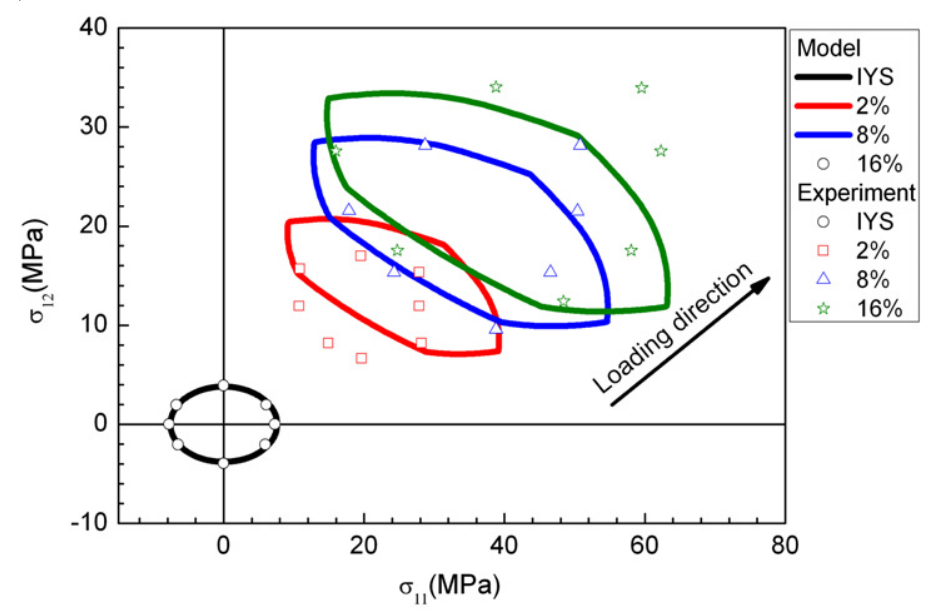

(b) annealed $1100 \mathrm{Al}$

Fig. 18. Comparison of the subsequent yield surfaces after combined tension-torsional loading between the prediction of the model and experimental data.

\subsection{The subsequent yield surfaces of non-proportional loading}

Using the same model parameters listed in Table 2, the subsequent yield surfaces of annealed $1100 \mathrm{Al}$ under non-proportional loading are predicted. The comparisons between predictions and experimental results are shown in Figs. 20-23. After $4 \%$ shear strain followed by tensile loading, the subsequent yield surfaces are shown at $2 \%, 8 \%$ and $16 \%$ tensile strains in Fig. 20. After $10 \%$ shear strain followed by tensile loading, the subsequent yield surfaces are shown at $2 \%$ and $8 \%$ tensile strains in Fig. 21. After 2\% tensile strain followed by torsional loading, the subsequent yield surfaces are shown at $4 \%$, $10 \%$ and $20 \%$ shear strains in Fig. 22 . After $8 \%$ tensile strain followed by torsional loading, the subsequent yield surfaces are shown at $4 \%$ and $10 \%$ shear strains in Fig. 23.

It can be seen from Figs. 20-23 that the subsequent yield surfaces after $4 \%$ and $10 \%$ shear strains followed by tensile loading are smaller as compared to those obtained under tensile loading, and those after $2 \%$ and $8 \%$ tensile strains followed by torsional loading are smaller than those obtained under torsional loading. The complex distortion of the yield surfaces is related to the activation and latent hardening of different slip components.

The subsequent yield surfaces show mixed cross effect with plastic deformation following loading path change. This can be explained by the different evolution tendencies and different influences on subsequent yield surface of kinematic hardening and isotropic hardening. As mentioned above, the kinematic hardening leads to negative cross effect, while the isotropic hardening results in positive cross effect. When two kinds of hardening both exist, whether the subsequent yield surfaces show positive or negative cross effect depends on the competition of two kinds of hardening. In the present model, the loading path change involves the change of active slip components, i.e. after the loading path change, the active slip components are different from those active in the previous loading stage. The shearing deformations of these newly active slip components are zero when loading path begins to change. According to the self hardening rule of kinematic part which is related to 


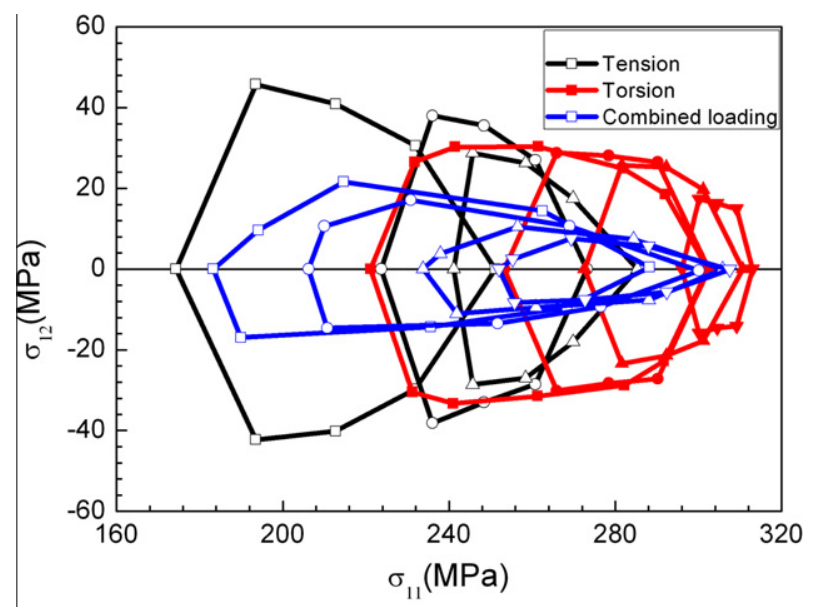

(a) $\mathrm{Al} 6061-\mathrm{T} 6511$

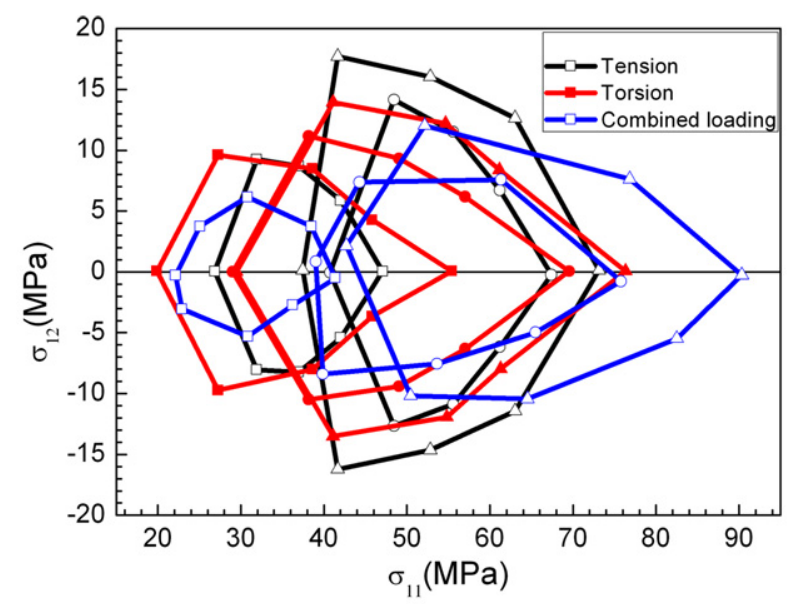

(b) annealed $1100 \mathrm{Al}$

Fig. 19. Comparison of the subsequent yield surfaces under three proportional loading paths.

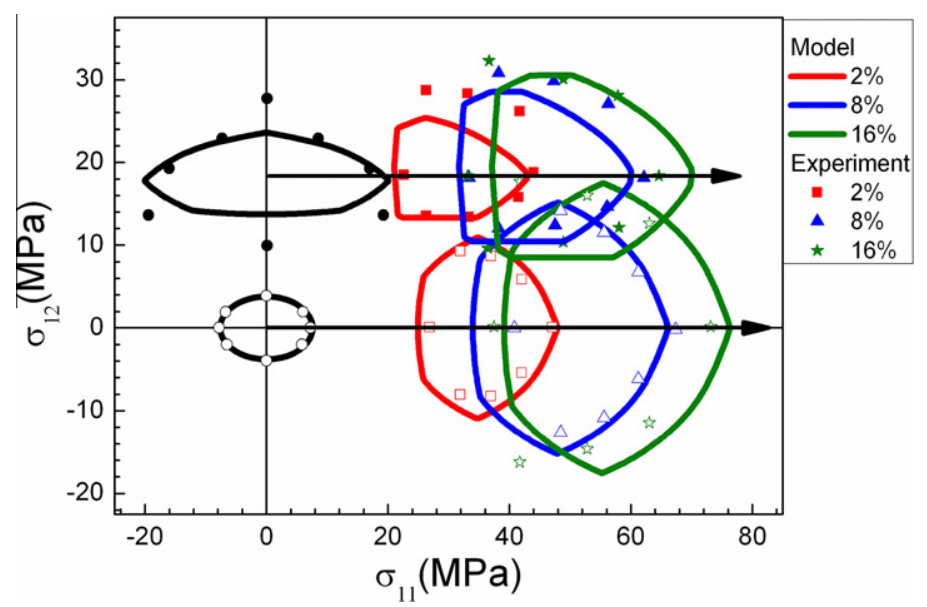

Fig. 20. Comparison of the subsequent yield surfaces of annealed $1100 \mathrm{Al}$ after tensile strain and after $4 \%$ shear strain followed by tensile loading between the prediction of the model and experimental data. 


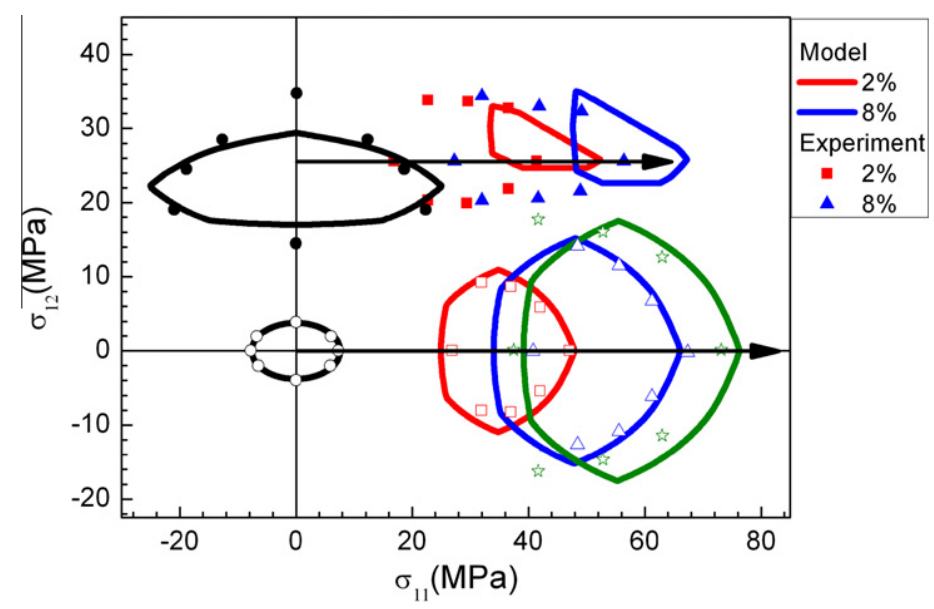

Fig. 21. Comparison of the subsequent yield surfaces of annealed $1100 \mathrm{Al}$ after tensile strain and after $10 \%$ shear strain followed by tensile loading between the prediction of the model and experimental data.

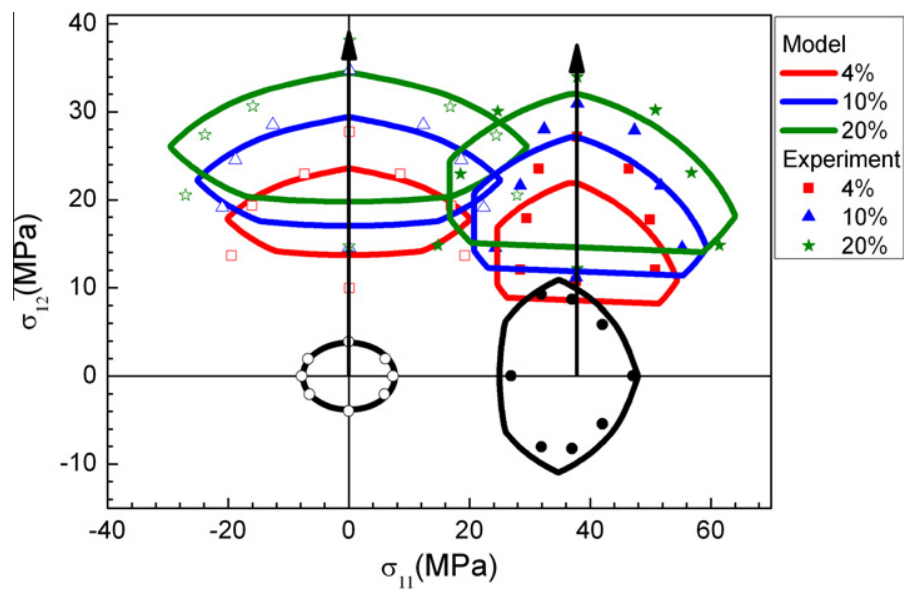

Fig. 22. Comparison of the subsequent yield surfaces of annealed $1100 \mathrm{Al}$ after shear strain and after $2 \%$ tensile strain followed by torsional loading between the prediction of the model and experimental data.

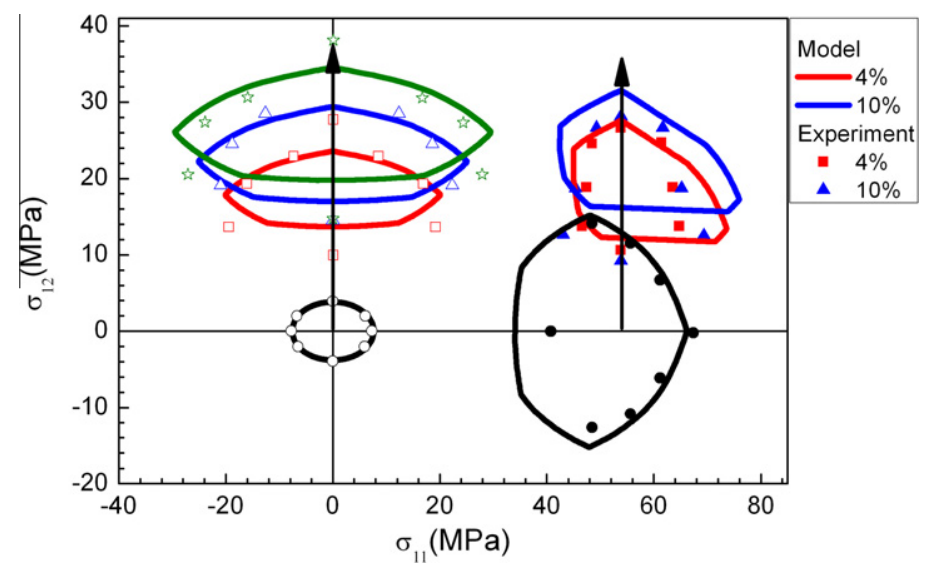

Fig. 23. Comparison of the subsequent yield surfaces of annealed $1100 \mathrm{Al}$ after shear strain and after $8 \%$ tensile strain followed by torsional loading between the prediction of the model and experimental data. 
the shearing deformations on the slip components by Eq. (60), the kinematic hardening increases suddenly when loading path changes. On the other hand, the isotropic hardening is related to the equivalent plastic strain by Eq. (58). When loading path changes as the experiment, the equivalent plastic strain increases continuously, so the isotropic hardening decreases continuously. Therefore, the kinematic hardening dominates the hardening rule at the beginning of the load path change, which makes the size of subsequent yield surface decreases. As load increases, kinematic hardening decreases quickly as the shearing deformations on the active slip components increase, while isotropic hardening decreases slower than kinematic hardening. Therefore, isotropic hardening exhibits its influences on the subsequent yield surfaces gradually. Once the isotropic hardening dominates the cross effect, the size of subsequent yield surfaces increases gradually. Therefore, the subsequent yield surfaces exhibit mixed cross effect in the case of non-proportional loading.

The agreement between predictions and experimental results on the subsequent yield surfaces under non-proportional loading demonstrates that the present model has the ability to deal with non-proportional loading due to the state variables on slip components (the positive and negative critical resolved shear stresses, $\tau_{+c r}^{(\alpha)}$ and $\tau_{-c r}^{(\alpha)}$ ). They are naturally different according to their different orientations and are related to the resolved shear stress, latent hardening or Bauschinger effect. Since the critical resolved shear stresses on all slip components determine the subsequent yield surface, the applied stress would create anisotropy for the material in a natural manner. Moreover, the evolution of the state variables records the loading history of material, which makes the proposed model have the potential to address the issue of complex loading.

One reason that there are a few differences between the predictions and experimental results is that the yield is defined by $10 \mu \varepsilon$ deviation from linearity in experiment, while the yield definition used in present paper is the absolutely proportional limit.

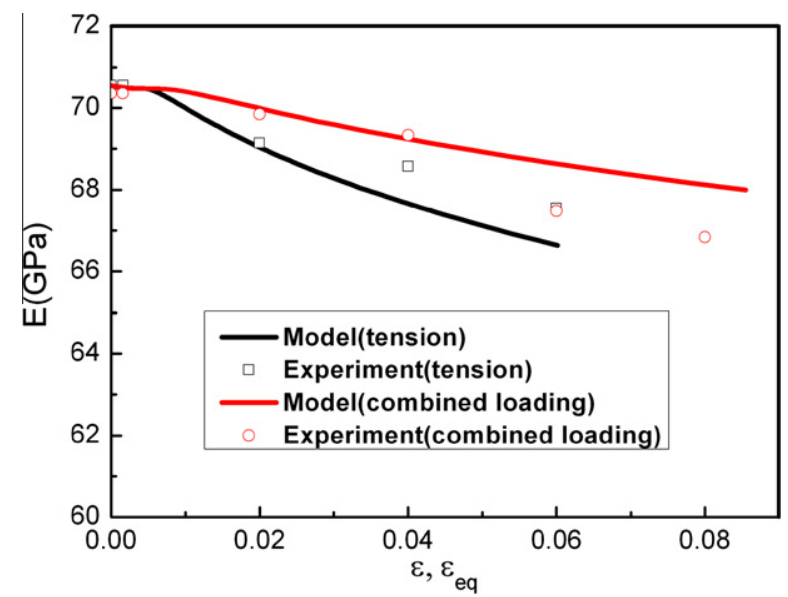

Fig. 24. The variation of Young's modulus of Al 6061-T6511 with finite plastic deformation.

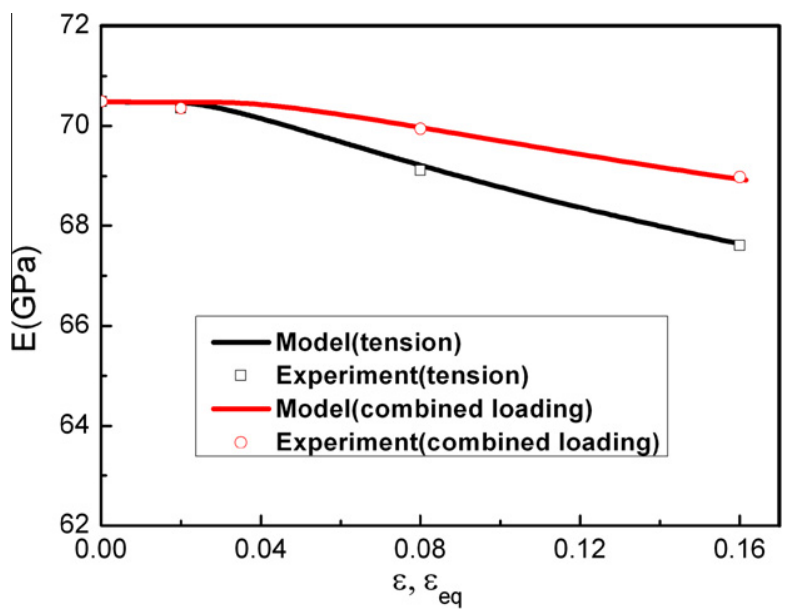

Fig. 25. The variation of Young's modulus of annealed $1100 \mathrm{Al}$ with finite plastic deformation. 


\subsection{The variation of elastic constants}

In experiment, the values of Young's and shear moduli within subsequent yield surfaces were also determined after each prestrain. The comparisons of elastic modulus evolution of Al 6061-T6511 and annealed $1100 \mathrm{Al}$ as a function of plastic deformation between simulations and experimental results are given in Fig. 24 and 25, respectively. It can be seen that the present model with damage defined on the spring-bundle component level has the ability to reflect the variation of elastic constants or macroscopic damage.

\section{Conclusions}

The present work has led to the following conclusions:

(1) A material model assembling with spring-bundle components, a cubage component and slip components is established, and corresponding elasto-plastic damage constitutive relation is derived under finite deformation. The iteration process and the calibration procedure to realize the constitutive relation are given.

(2) The numerical simulations are performed under tension, torsion, and combined tension-torsion proportional loading conditions for two kinds of aluminum alloys. Based on the Bauschinger effect and latent hardening of the slip components, the subsequent yield surface can be described that the rear part deflates and the forward part inflates so that the subsequent yield surface has a sharp front and a blunt rear. For low work hardening aluminum alloy Al 6061-T6511, the size of subsequent yield surface decreases because of the combined effect of latent hardening and Bauschinger effect. For high work hardening aluminum alloy annealed $1100 \mathrm{Al}$, the size of subsequent yield surface increases because of isotropic hardening. Further, the difference between the prediction results and the experimental results are investigated in terms of the equivalence of three proportional loading paths.

(3) The numerical simulations are performed under non-proportional loading conditions for annealed $1100 \mathrm{Al}$. The subsequent yield surfaces after shear strains followed by tensile loading and those after tensile strains followed by torsional loading show mixed cross effect because of the different evolution laws of kinematic hardening and isotropic hardening when loading path change. The competition between kinematic hardening and isotropic hardening determines whether the subsequent yield surfaces show positive or negative cross effect. Kinematic hardening which is related to the shearing deformation on active slip components increases suddenly when loading path change and decreases quickly as load continues to increase, while isotropic hardening which is related to the equivalent plastic deformation decreases continuously. Therefore, at the initial stage of loading path change, the kinematic hardening dominates the hardening rule, and the subsequent yield surfaces show negative cross effect. As load increases, the isotropic hardening exhibits its influences on subsequent yield surfaces gradually, and the subsequent yield surfaces show positive cross effect.

(4) The state variables on slip components (positive and negative critical resolved shear stresses) which change according to hardening or Bauschinger effect are different according to the different orientations of slip components, which reflects the plasticity induced anisotropy. Moreover, the state variables are related to the applied stress and can reflect the influence of the loading path. The prediction results under non-proportional loading conditions demonstrate that the present model based on the state variables defined on the slip components has the ability to deal with the nonproportional loading problem.

(5) As an elasto-plastic damage constitutive model, the present model can also reflect the variation of elastic constants based on the damage defined on the spring-bundle components. Moreover, similar to the slip components, the state variables on spring-bundle components (maximum and minimum deformations experienced by spring-bundle components) are different according to the different orientations of spring-bundle components and related to the elastic deformation, which reflects the damage induced anisotropy. Therefore, the model has the ability to address the issue of non-proportional loading with emphasis on the description of anisotropy induced by damage as well as plastic deformation.

(6) The proposed model incorporates the important feature of crystal plasticity, and the parameters in the present model have their physical meaning. Moreover, the parameters are the same in all loading cases, i.e. it is not necessary to recalibrate the model parameters under a different loading condition.

\section{Acknowledgements}

The authors thank Prof. Khan, A.S. for providing the experimental data of Al 6061-T6511. This work was supported by the National Natural Science Foundation of China under Grant Nos. 10572140 and 11021262.

\section{References}

Asaro, R.J., 1983. Micromechanics of crystals and polycrystals. Adv. Appl. Mech. 23, 1-115.

Ascione, L., Olivito, R., Spadea, G., 1982. An experimental study on subsequent yield surfaces for metals. Mater. Struct. 15, 21-26. 
Batdorf, S.B., Budiansky, B., 1949. A mathematical theory of plasticity based on the concept of slip. NACA TN 1871.

Brünig, M., 2003. An anisotropic ductile damage model based on irreversible thermodynamics. Int. J. Plast. 19, 1679-1713.

Chiang, D.Y., Su, K.H., Liao, C.H., 2002. A study on subsequent yield surface based on the distributed-element model. Int. J. Plast. 18, 51-70.

Deng, S.C., Liu, J.X., Zhang, J., Liang, N.G., 2006. Component assembling model and its application to quasi-brittle damage. Theor. Appl. Fract. Mech. 46, 232242.

Deng, S.C., Liu, J.X., Liang, N.G., Zhang, J., 2007. Validation of component assembly model and extension to plasticity. Theor. Appl. Fract. Mech. 47, 244259.

Ericksen, J.L., 1984. The Cauchy and Born Hypotheses for Crystals. Phase Transformations and Material Instabilities in Solids. Academic Press, New York. Feigenbaum, H.P., Dafalias, Y.F., 2007. Directional distortional hardening in metal plasticity within thermodynamics. Int. J. Solids Struct. 44, 7526-7542.

Feigenbaum, H.P., Dafalias, Y.F., 2008. Simple model for directional distortional hardening in metal plasticity within thermodynamics. J. Eng. Mech. 134, 730-738.

Francois, M., 2001. A plasticity model with yield surface distortion for non proportional loading. Int. J. Plast. 17, 703-717.

Fu, Q., Liu, F., Zhang, J., Liang, N.G., 2010. A physically motivated model for the evolution of subsequent yield surfaces. Chin. J. Theor. Appl. Mech. 42, 880888 (in Chinese).

Gil, C.M., Lissenden, C.J., Lerch, B.A., 1999. Yield of inconel 718 by axial-torsional loading at temperatures up to $649{ }^{\circ} \mathrm{C}$. J. Test. Eval. $27,327-336$.

Gupta, N.K., Meyers, A., 1992. Considerations of translated stress deviators in describing yield surfaces. Int. J. Plast. 8, 729-740.

Helling, D., Miller, A., 1987. The incorporation of yield surface distortion into a unified constitutive model, Part 1: Equation development. Acta Mech. 69, 923.

Helling, D., Miller, A., 1988. The incorporation of yield surface distortion into a unified constitutive model, Part 2: Predictive capabilities. Acta Mech. 72, 3953.

Helling, D.E., Miller, A.K., Stout, M.G., 1986. An experimental investigation of the yield loci of 1100-0 aluminum, 70:30 brass, and an overaged 2024 aluminum alloy after various prestrains. J. Eng. Mater. Technol., Trans. ASME 108, 313-320.

Ishikawa, H., 1997. Subsequent yield surface probed from its current center. Int. J. Plast. 13, 533-549.

Ivey, H.J., 1961. Plastic stress-strain relations and yield surfaces for aluminium alloys. J. Mech. Eng. Sci. 3, 15-31.

Kalidindi, S.R., Schoenfeld, S.E., 2000. On the prediction of yield surfaces by the crystal plasticity models for fcc polycrystals. Mater. Sci. Eng. A - Struct. Mater. Prop. Microstruct. Process. 293, 120-129.

Khan, A.S., Cheng, P., 1996. An anisotropic elastic-plastic constitutive model for single and polycrystalline metals I - theoretical developments. Int. J. Plast. $12,147-162$.

Khan, A.S., Wang, X.W., 1988. On non-proportional infinitesimal plastic-deformation after finite plastic prestraining and partial unloading. J. Mech. Phys. Solids 36, 519-535.

Khan, A.S., Wang, X.W., 1993. An experimental study on subsequent yield surface after finite shear prestraining. Int. J. Plast. 9, 889-905.

Khan, A.S., Kazmi, R., Pandey, A., Stoughton, T., 2009. Evolution of subsequent yield surfaces and elastic constants with finite plastic deformation, Part-I: A very low work hardening aluminum alloy (Al6061-T6511). Int. J. Plast. 25, 1611-1625.

Khan, A.S., Pandey, A., Stoughton, T., 2010a. Evolution of subsequent yield surfaces and elastic constants with finite plastic deformation, Part II: A very high work hardening aluminum alloy (annealed $1100 \mathrm{Al}$ ). Int. J. Plast. 26, 1421-1431.

Khan, A.S., Pandey, A., Stoughton, T., 2010b. Evolution of subsequent yield surfaces and elastic constants with finite plastic deformation, Part III: Yield surface in tension-tension stress space (Al 6061-T 6511 and annealed $1100 \mathrm{Al}$ ).. Int. J. Plast. 26, 1432-1441.

Kowalsky, U., Ahrens, H., Dinkler, D., 1999. Distorted yield surfaces-modelling by higher order anisotropic hardening tensors. Comput. Mater. Sci. 16, 81-88.

Krajcinovic, D., 2000. Damage mechanics: accomplishments, trends and needs. Int. J. Solids Struct. 37, 267-277.

Liang, N.G., Liu, H.Q., Wang, T.C., 1998. A meso elastoplastic constitutive model for polycrystalline metals based on equivalent slip systems with latent hardening. Sci. China, Ser. A 41, 887-896.

Liu, F., Fu, Q., Chen, C., Liang, N., 2010. An elasto-plastic damage constitutive theory based on pair functional potentials and slip mechanism. Chin. J. Aeronaut. 23, 686-697.

Lu, T.J., Chow, C.L., 1990. On constitutive equations of inelastic solids with anisotropic damage. Theor. Appl. Fract. Mech. 14, 187-218.

Mair, W.M., Pugh, H.L.D., 1964. Effect of pre-strain on yield surfaces in copper. J. Mech. Eng. Sci. 6. 150-\&.

Martin, J.B., 1975. Plasticity: Fundamentals and General Results. The MIT Press, Cambridge.

Michael, M.J., Findley, W.N., 1976. An historical perspective of yield surface investigations for metals. Int. J. Non-Linear Mech. 11, 59-82.

Mollica, F., Srinivasa, A.R., 2002. A general framework for generating convex yield surfaces for anisotropic metals. Acta Mech. 154, 61-84.

Moreton, D.N., Moffat, D.G., Parkinson, D.B., 1981. The yield surface behavior of pressure-vessel steels. J. Strain Anal. Eng. Des. 16, $127-136$.

Murakami, S., 1988. Mechanical modeling of material damage. J. Appl. Mech. 55, 280-286.

Naghdi, P.M., Essengurg, F., Koff, W., 1958. An experimental study of initial and subsequent yield surfaces in plasticity. J. Appl. Mech. 25, 201-209.

Ortiz, M., Popov, P.E., 1983. Distortional hardening rules for metal plasticity. J. Eng. Mech. 109, 1042-4057.

Phillips, A., Das, P.K., 1985. Yield surfaces and loading surfaces of aluminum and brass: an experimental investigation at room and elevated temperatures. Int. J. Plast. 1, 89-109.

Phillips, A., Ricciuti, M., 1976. Fundamental experiments in plasticity and creep of aluminum-extension of previous results. Int. J. Solids Struct. 12, 159171.

Rees, D.W.A., 1984. An examination of yield surface distortion and translation. Acta Mech. 52, 15-40.

Rousselier, G., Barlat, F., Yoon, J.W., 2009. A novel approach for anisotropic hardening modeling. Part I: Theory and its application to finite element analysis of deep drawing. Int. J. Plast. 25, 2383-2409.

Shiratori, E., Ikegami, K., Kaneko, K., 1973. The influence of the Bauschinger effect on the subsequent yield condition. Bull. JSME 16, $1482-1493$.

Simo, J.C., Ju, J.W., 1987. Strain- and stress-based continuum damage models - I. Formulation. Int. J. Solids Struct. 23, 821-840.

Souchet, R., 2005. Equivalence principles in continuum damage mechanics. Int. J. Eng Sci. 43, 1313-1322.

Stout, M.G., Martin, P.L., Helling, D.E., Canova, G.R., 1985. Multiaxial yield behavior of 1100 aluminum following various magnitudes of prestrain. Int. J. Plast. $1,163-174$.

Suprun, A.N., 2006. A constitutive model with three plastic constants: the description of anisotropic workhardening. Int. J. Plast. 22, $1217-1233$.

Taylor, G.I., 1934. The mechanism of plastic deformation of crystals, Part I - Theoretical. Proc. R. Soc. Lond. Ser. A 145, 362-387.

Tozawa, Y., 1978. Plastic deformation behavior under conditions of combined stress. In: Koistinen, D.P., Wang, N.-M. (Eds.), Mechanics of Sheet Metal Forming. Plenum Press, New York, London.

Voyiadjis, G.Z., Foroozesh, M., 1990. Anisotropic distortional yield model. J. Appl. Mech. 57, 537-547.

Voyiadjis, G.Z., Park, T., 1999. The kinematics of damage for finite-strain elasto-plastic solids. Int. J. Eng. Sci. 37, 803-830.

Wang, T.C., 1991. Macro slip theory of plasticity for polycrystalline solids. Acta Mech. Sin. 7, 323-334.

Wegener, K., Schlegel, M., 1996. Suitability of yield functions for the approximation of subsequent yield surfaces. Int. J. Plast. 12, 1151-1177.

Williams, J., Svensson, N., 1970. Effect of tensile prestrain on the yield locus of 1100-f aluminium. J. Strain Anal. Eng. Des. 5, 128-139.

Williams, J., Svensson, N., 1971. Effect of torsional prestrain on the yield locus of 1100-F aluminium. J. Strain Anal. Eng. Des. 6, 263-272.

Wu, H.-C., 2002. Anisotropic plasticity for sheet metals using the concept of combined isotropic-kinematic hardening. Int. J. Plast. 18, $1661-1682$.

Wu, H.-C., 2003a. On finite plastic deformation of anisotropic metallic materials. Int. J. Plast. 19, 91-119.

Wu, H.C., 2003b. On finite plastic deformation of anisotropic metallic materials. Int. J. Plast. 19, 91-119.

Wu, H.C., Yeh, W.C., 1991. On the experimental determination of yield surfaces and some results of annealed 304 stainless steel. Int. J. Plast. 7, 803-826. 
Wu, H.C., Lu, J.K., Pan, W.F., 1995. Some observations on yield surfaces for 304 stainless steel at large prestrain. J. Appl. Mech., Trans. ASME 62, $626-632$.

Yeh, W.-C., Lin, H.-Y., 2006a. An endochronic model of yield surface accounting for deformation induced anisotropy. Int. J. Plast. 22, 16-38.

Yeh, W.C., Lin, H.Y., 2006b. An endochronic model of yield surface accounting for deformation induced anisotropy. Int. J. Plast. 22, 16-38.

Zattarin, P., Lipinski, P., Rosochowski, A., 2004. Numerical study of the influence of microstructure on subsequent yield surfaces of polycrystalline materials. Int. J. Mech. Sci. 46, 1377-1398.

Zhang, J., Liang, N.G., Deng, S.C., Liu, J.X., Liu, X.Y., Fu, Q., 2008. Study of the damage-induced anisotropy of quasi-brittle materials using the component assembling model. Int. J. Damage Mech. 17, 197-221. 